\title{
OSTEO-ARTHROSIS
}

\section{PREVALENCE IN THE POPULATION AND RELATIONSHIP BETWEEN SYMPTOMS AND $X$-RAY CHANGES}

\author{
BY \\ J. S. LAWRENCE, J. M. BREMNER, AND F. BIER \\ From the Arthritis and Rheumatism Council Field Unit and the Rheumatism Research Centre, \\ University of Manchester
}

From a study of rheumatic complaints in an urban population (Kellgren, Lawrence, and Aitken-Swan, 1953), it was concluded that osteo-arthrosis was the most frequent cause of loss of work due to rheumatic disease in females and that it came third as a cause of such incapacity in males. Anderson, Duthie, and Moody (1962) and Anderson and Duthie (1963) have since reached similar conclusions in groups of miners and dock-workers. The relative importance of osteo-arthrosis was confirmed by Mason (1956), who found it to be by far the largest diagnostic group among patients attending a physiotherapy department. In an $x$-ray survey of a random sample of the Leigh population aged 55 to 64 years, it was found that 83 per cent. of males and 87 per cent. of females had osteo-arthrosis in one joint or other and that the condition was present in four or more joints in 29 per cent. of males and 47 per cent. of females (Kellgren and Lawrence, 1958). In a later study in which samples of the population from age 15 were examined, it was observed that both the number of joints involved and the severity of the disease as determined radiologically was similar in males and females up to age 54 , but that the disease thereafter became more severe and more generalized in females (Kellgren, Lawrence, and Bier, 1963). The joint pattern was also similar in males and females in the younger age groups but changed after age 55, when the distal and proximal interphalangeal joints of the fingers, the metacarpophalangeal, and the 1st carpometacarpal joints were more frequently affected in females. The wrists and hips, on the other hand, were more commonly affected in males.

But, although osteo-arthrosis occurs with considerable frequency in population samples and is almost universal in the older age groups, mere prevalence statistics give little indication of its importance to the community whether as a cause of suffering or of economic loss. In a survey of coalmining and other occupational groups in which routine $x$-rays of the knees were taken, it was found that only 24 per cent. of men with definite radiological evidence of osteo-arthrosis gave a history of pain in the knees. Of those without radiological evidence, 8 per cent. gave such a history (Kellgren and Lawrence, 1952).

The reasons for this low complaint rate and the factors which determine the occurrence of symptoms are by no means clear. A climatic influence has been suggested. Thus, in a survey of rheumatic complaints in Leigh, complaints attributed to osteoarthrosis were rather more frequent in those working in cold or wet conditions than in those in a more equable environment (Kellgren and others, 1953). Coal-miners working in a wet seam lost more work from knee symptoms than those in dry seams, though the radiological changes were no different (Lawrence, 1955). Moreover, textile and foundry employees working in a warm environment have been found to have less incapacity than the rest of the population despite a high prevalence of radiological change (Lawrence, 1961 ; Lawrence, Dingwall-Fordyce, and Molyneux, 1966). Duthie, on the other hand, has found no evidence of any climatic influence.

Cobb, Merchant, and Rubin (1957) observed that pain and swelling in osteo-arthrosic joints were seldom found without morning stiffness and that persons with $x$-ray evidence of osteo-arthrosis but no morning stiffness had only a small chance of having pain at the relevant site. They suggested that most of the symptoms of osteo-arthrosis are at least in part due to a rheumatoid component.

Kellgren (1961) suggested that some of the painful episodes in this condition may be due to a ligamentous sprain or traumatic synovitis following minor injury to a joint rendered unstable by the disease.

In the present paper the prevalence of osteoarthrosis in populations in Northern England is reported and its relationship to symptoms in the region of the joints investigated. 
Material and Completion Rate

The present study is based on the Leigh random sample and the Wensleydale area sample. Papers by Lawrence and Bennett (1960) and by Bremner (1961) should be consulted for details of the method of sampling and of the clinical and $x$-ray examinations. Since these surveys were reported certain additions have been made. In Leigh a further sample of persons aged 65 and over has been examined to increase the numbers available in this age group which tends to be low in a true random sample. In Wensleydale the completion rate has been raised by examining at home a further sample of those who were unable to attend for $x$ ray in the original survey. The number from the combined Leigh-Wensleydale sample who have been $x$ rayed at the present time is shown in Table I. In both sexes 86 per cent. had one or more $x$ rays. The proportion varied from 80 per cent. in the older females to 91 per cent. in females aged 25 to 34 . In males it varied from 83 per cent. in the youngest age group to 90 per cent. in the 25 to 34 age group. Those who had only a clinical examination and blood test have not been included in the Table.

$x$ rays were taken of the hands, feet, and cervical spine, and, from the age of 35 onwards, the lumbar spine and knees. The hips were included in those aged 55 and over. Thus it was possible to investigate the relationship of $x$ ray changes to symptoms on a wide variety of joints. Bias was avoided by reading the $x$ rays without knowledge of the clinical features. In some instances special $x$ rays were taken in addition to those routinely studied. These included such joints as the elbow, shoulder, and dorsal spine, or joints normally examined only at a higher age. These were counted in the number of joint groups affected in Table IIA but not in IIB or any of the subsequent Tables. The joint group is defined as all joints of one type, e.g. the distal interphalangeal joints of the fingers. The carpometacarpal and intracarpal joints are included in one group. Only those persons in the sample who had adequate $x$ rays to determine the number of joint groups affected were included in the study. Even so the reader will find that the total given as having been $x$ rayed varies in different Tables, as number of individuals refused $x$ ray of one part of theip body.

Respondents were asked whether they suffered frome rheumatic pain and if so whether it had been presento in the past week and whether it had resulted in loss of work at any time. In addition, during the physicas? examination of each joint, the respondent was aske whether he had ever had pain in that joint. In this waষ symptoms which had been forgotten during the initian history-taking were frequently remembered and recorded It is likely that, apart from those pains giving rise to loss of work, most of these symptoms were not such as ton lead to a consultation with the family doctor. In an earlier study it was found that only some 50 per cent. of persons with symptoms attributable to osteo-arthrosis consulted their doctor about it (Kellgren and others 1953).

Prevalence
The prevalence of osteo-arthrosis in this popula

The prevalence of osteo-arthrosis in this popula (opposite and overleaf). Adequate data were avai $\vec{E}$ able on 1,098 males and 1,198 females.

As this sample is selected so as to contain more than the true proportion of the population in the older age groups, prevalence based on the total numberfo in all age groups examined would be meaningess Instead the percentage prevalences in the various age groups were added together and divided by the nun of age groups to obtain an "unweighted mean" prezao lence. The standard population thus adopted by 4 omits the youngest age groups, under 15 years, as no interesting in our problem, uses decennial age groups from 15-64 years, and also includes a single age group of 65 years and over: within this range, it gives somewhat more than their true weight to the older age groups but the overall difference is not great in a population of the present age distribution in the United Kingdom as wilf

TABLE I

COMPLETION RATE IN THE COMBINED LEIGH AND WENSLEYDALE ENLARGED SAMPLES IN 1963, BY AGE AND SEX

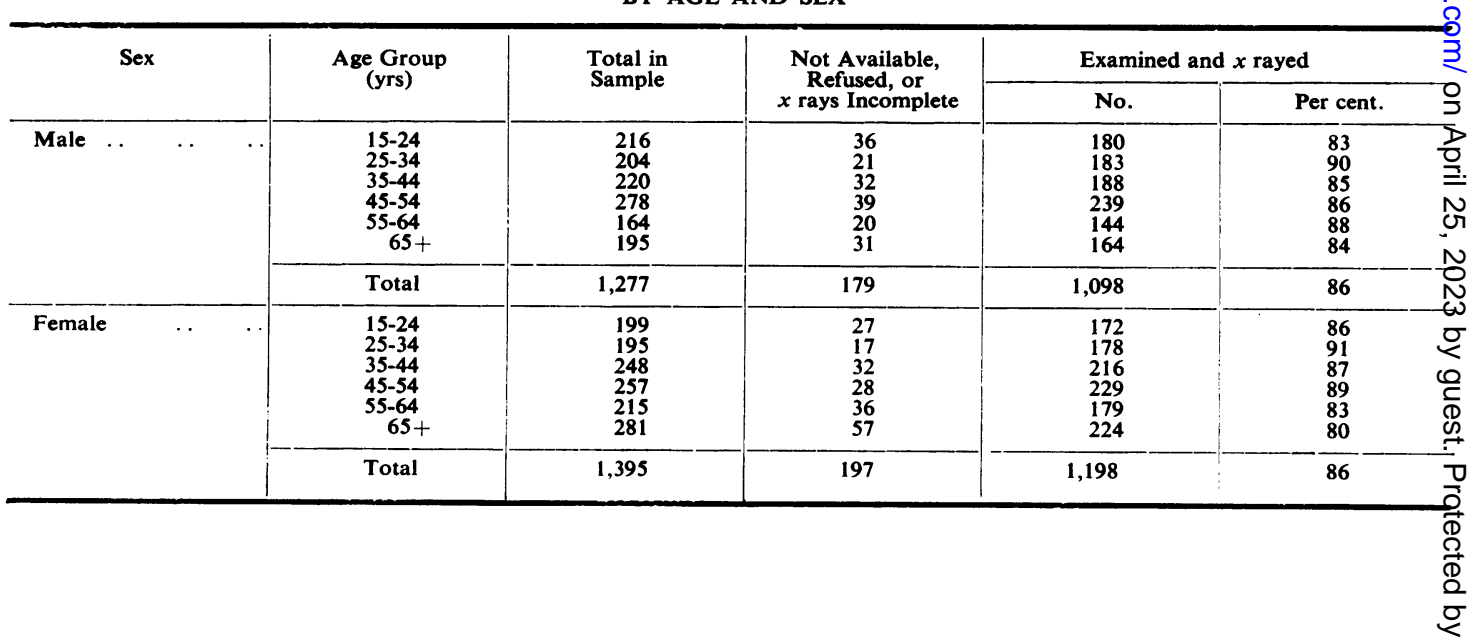



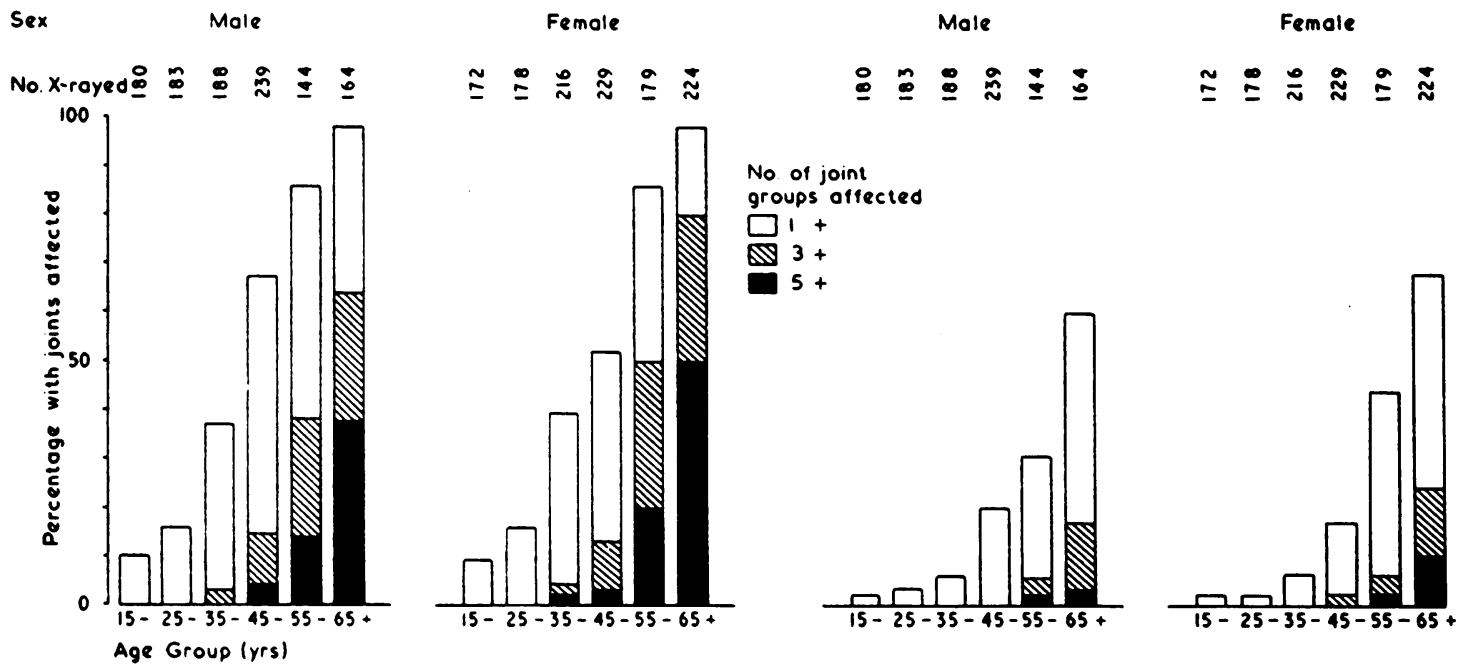

Fig. 1.-Prevalence of grades of osteo-arthrosis in combined Leigh and Wensleydale samples, by sex, age, and number of joints affected.

TABLE IIA

AGE AND SEX DISTRIBUTION OF RADIOLOGICAL OSTEO-ARTHROSIS IN THE LEIGH AND WENSLEYDALE ENLARGED SAMPLES

Number of joint groups affected of those $x$ rayed routinely and specially, by two grades of severity

\begin{tabular}{|c|c|c|c|c|c|c|c|c|c|c|c|c|c|c|}
\hline \multirow{4}{*}{ Sex } & \multirow{4}{*}{$\underset{\text { (yrs) }}{\text { Age }}$} & \multirow{4}{*}{$\begin{array}{l}\text { Total } \\
\text { No. of } \\
\text { Persons } \\
x \text { rayed }\end{array}$} & \multicolumn{12}{|c|}{ Number of Joint Groups with Osteo-arthrosis } \\
\hline & & & \multicolumn{3}{|c|}{ Grade $2-4$} & \multicolumn{3}{|c|}{ Grade 3-4 } & \multicolumn{3}{|c|}{ Grade 2-4 } & \multicolumn{3}{|c|}{ Grade 3-4 } \\
\hline & & & $1+$ & $3+$ & $5+$ & $1+$ & $3+$ & $5+$ & $1+$ & $3+$ & $5+$ & $1+$ & $3+$ & $5+$ \\
\hline & & & \multicolumn{6}{|c|}{ Number of Persons } & \multicolumn{6}{|c|}{ Per cent. of Total $x$ rayed } \\
\hline \multirow[t]{3}{*}{ Male } & $\begin{array}{r}15-24 \\
25-34 \\
35-44 \\
45-54 \\
55-64 \\
65+\end{array}$ & $\begin{array}{l}180 \\
183 \\
188 \\
239 \\
144 \\
164\end{array}$ & $\begin{array}{r}17 \\
30 \\
72 \\
159 \\
124 \\
159\end{array}$ & $\begin{array}{r}0 \\
0 \\
4 \\
33 \\
54 \\
104\end{array}$ & $\begin{array}{r}0 \\
0 \\
0 \\
6 \\
18 \\
61\end{array}$ & $\begin{array}{r}2 \\
4 \\
11 \\
46 \\
43 \\
95\end{array}$ & $\begin{array}{r}0 \\
0 \\
0 \\
0 \\
6 \\
26\end{array}$ & $\begin{array}{l}0 \\
0 \\
0 \\
0 \\
1 \\
4\end{array}$ & $\begin{array}{r}9 \cdot 4 \\
16 \cdot 4 \\
38 \cdot 3 \\
66 \cdot 5 \\
86 \cdot 1 \\
97 \cdot 0\end{array}$ & $\begin{array}{c}0 \\
0 \\
2 \cdot 1 \\
13 \cdot 8 \\
37 \cdot 5 \\
63 \cdot 4\end{array}$ & $\begin{array}{c}0 \\
0 \\
0 \\
2 \cdot 5 \\
12 \cdot 5 \\
37 \cdot 2\end{array}$ & $\begin{array}{r}1 \cdot 1 \\
2 \cdot 2 \\
5.9 \\
19 \cdot 2 \\
29.9 \\
57 \cdot 9\end{array}$ & $\begin{array}{c}0 \\
0 \\
0 \\
0 \\
4 \cdot 2 \\
15 \cdot 8\end{array}$ & $\begin{array}{l}0 \\
0 \\
0 \\
0 \\
0 \cdot 7 \\
2 \cdot 4\end{array}$ \\
\hline & Total 15+ & 1098 & 561 & 195 & 85 & 201 & 32 & 5 & $51 \cdot 1$ & $17 \cdot 8$ & $7 \cdot 7$ & $18 \cdot 3$ & $2 \cdot 9$ & 0.5 \\
\hline & \multicolumn{2}{|c|}{$\begin{array}{l}\text { Unweighted Mean } \\
\text { Weighted Mean }\end{array}$} & & & & & & & $\begin{array}{l}52 \cdot 3 \\
49 \cdot 0\end{array}$ & $\begin{array}{l}19 \cdot 5 \\
16 \cdot 5\end{array}$ & $\begin{array}{l}8 \cdot 7 \\
7 \cdot 0\end{array}$ & $\begin{array}{l}19 \cdot 4 \\
16 \cdot 9\end{array}$ & $\begin{array}{l}3 \cdot 3 \\
2 \cdot 6\end{array}$ & $\begin{array}{l}0.5 \\
0.4\end{array}$ \\
\hline \multirow[t]{3}{*}{ Female } & $\begin{array}{r}15-24 \\
25-34 \\
35-44 \\
45-54 \\
55-64 \\
65+\end{array}$ & $\begin{array}{l}172 \\
178 \\
216 \\
229 \\
179 \\
224\end{array}$ & $\begin{array}{r}13 \\
26 \\
84 \\
140 \\
154 \\
218\end{array}$ & $\begin{array}{r}0 \\
0 \\
6 \\
28 \\
90 \\
178\end{array}$ & $\begin{array}{r}0 \\
0 \\
2 \\
5 \\
34 \\
109\end{array}$ & $\begin{array}{r}1 \\
1 \\
12 \\
36 \\
78 \\
152\end{array}$ & $\begin{array}{r}0 \\
0 \\
0 \\
3 \\
10 \\
54\end{array}$ & $\begin{array}{r}0 \\
0 \\
0 \\
2 \\
2 \\
19\end{array}$ & $\begin{array}{r}7 \cdot 6 \\
14 \cdot 6 \\
38 \cdot 9 \\
61 \cdot 2 \\
86 \cdot 1 \\
97 \cdot 3\end{array}$ & $\begin{array}{c}0 \\
0 \\
2 \cdot 8 \\
12 \cdot 2 \\
50 \cdot 3 \\
79 \cdot 5\end{array}$ & $\begin{array}{c}0 \\
0 \\
0 \cdot 9 \\
2 \cdot 2 \\
19 \cdot 0 \\
48 \cdot 7\end{array}$ & $\begin{array}{r}0.6 \\
0.6 \\
5.6 \\
15.7 \\
43.6 \\
67.9\end{array}$ & $\begin{array}{l}0 \\
0 \\
0 \\
1 \cdot 3 \\
5 \cdot 6 \\
24 \cdot 1\end{array}$ & $\begin{array}{l}0 \\
0 \\
0 \\
0 \cdot 9 \\
1 \cdot 1 \\
8 \cdot 5\end{array}$ \\
\hline & Total $15+$ & 1198 & 635 & 302 & 150 & 280 & 67 & 23 & $53 \cdot 1$ & $25 \cdot 2$ & $12 \cdot 5$ & $23 \cdot 4$ & $5 \cdot 6$ & 1.9 \\
\hline & \multicolumn{2}{|c|}{$\begin{array}{l}\text { Unweighted Mean } \\
\text { Weighted Mean }\end{array}$} & & & & & & & $\begin{array}{l}51 \cdot 0 \\
51.9\end{array}$ & $\begin{array}{l}24 \cdot 1 \\
24 \cdot 9\end{array}$ & $\begin{array}{l}11.9 \\
12.3\end{array}$ & $\begin{array}{l}22 \cdot 3 \\
23 \cdot 0\end{array}$ & $\begin{array}{l}5 \cdot 2 \\
5 \cdot 5\end{array}$ & $\begin{array}{l}1.8 \\
1.9\end{array}$ \\
\hline
\end{tabular}

Note: The weighted means are based on the age distribution of males and females in England and Wales according to the Population Census of 1961 . 
TABLE IIB

AGE AND SEX DISTRIBUTION OF RADIOLOGICAL OSTEO-ARTHROSIS IN THE LEIGH AND WENSLEYDALE ENLARGED SAMPLES

Number of joint groups affected of those $x$ rayed routinely, by two grades of severity

\begin{tabular}{|c|c|c|c|c|c|c|c|c|c|c|c|c|c|c|c|}
\hline \multirow{4}{*}{$\begin{array}{c}\text { No. of } \\
\text { Joint Groups } \\
x \text { rayed } \\
\text { Routinely }\end{array}$} & \multirow{4}{*}{ Sex } & \multirow{4}{*}{$\begin{array}{c}\text { Age } \\
\text { Group } \\
\text { (yrs) }\end{array}$} & \multirow{4}{*}{$\begin{array}{l}\text { Total } \\
\text { No. of } \\
\text { Persons } \\
x \text { rayed }\end{array}$} & \multicolumn{12}{|c|}{ Number of Joint Groups with Osteo-arthrosis } \\
\hline & & & & \multicolumn{3}{|c|}{ Grade 2-4 } & \multicolumn{3}{|c|}{ Grade 3-4 } & \multicolumn{3}{|c|}{ Grade $2-4$} & \multicolumn{3}{|c|}{ Grade 3-4 } \\
\hline & & & & $1+$ & $3+$ & $5+$ & $1+$ & $3+$ & $5+$ & $1+$ & $3+$ & $5+$ & $1+$ & $3+$ & $5+$ \\
\hline & & & & \multicolumn{6}{|c|}{ Number of Persons } & \multicolumn{6}{|c|}{ Per cent. of Total $x$ rayed } \\
\hline \multirow[t]{6}{*}{9} & \multirow[t]{3}{*}{ Male } & $\begin{array}{l}15-24 \\
25-34 \\
35-44 \\
45-54 \\
55-64 \\
65+\end{array}$ & $\begin{array}{l}179 \\
182 \\
184 \\
236 \\
142 \\
162\end{array}$ & $\begin{array}{r}13 \\
27 \\
49 \\
138 \\
115 \\
145\end{array}$ & $\begin{array}{r}0 \\
0 \\
1 \\
19 \\
33 \\
82\end{array}$ & $\begin{array}{r}0 \\
0 \\
0 \\
2 \\
6 \\
39\end{array}$ & $\begin{array}{r}1 \\
5 \\
5 \\
34 \\
33 \\
76\end{array}$ & $\begin{array}{r}0 \\
0 \\
0 \\
0 \\
4 \\
19\end{array}$ & $\begin{array}{l}0 \\
0 \\
0 \\
0 \\
0 \\
2\end{array}$ & $\begin{array}{r}7 \cdot 3 \\
14 \cdot 8 \\
26 \cdot 6 \\
58 \cdot 5 \\
81 \cdot 0 \\
89 \cdot 5\end{array}$ & $\begin{array}{l}0 \\
0 \\
0 \cdot 5 \\
8 \cdot 1 \\
23 \cdot 2 \\
50 \cdot 6\end{array}$ & $\begin{array}{l}0 \\
0 \\
0 \\
0 \cdot 8 \\
4 \cdot 2 \\
24 \cdot 1\end{array}$ & $\begin{array}{r}0 \cdot 6 \\
2 \cdot 8 \\
2 \cdot 7 \\
14 \cdot 4 \\
23 \cdot 2 \\
46 \cdot 9\end{array}$ & \begin{tabular}{c|}
0 \\
0 \\
0 \\
0 \\
$2 \cdot 8$ \\
$11 \cdot 7$
\end{tabular} & $\begin{array}{l}0 \\
0 \\
0 \\
0 \\
0 \\
1 \cdot 2\end{array}$ \\
\hline & & Total $15+$ & 1085 & 487 & 135 & 47 & 154 & 23 & 2 & $44 \cdot 9$ & $12 \cdot 4$ & $4 \cdot 3$ & $14 \cdot 2$ & $2 \cdot 1$ & $0 \cdot 2$ \\
\hline & & \multicolumn{7}{|c|}{ Unweighted Mean } & & $46 \cdot 2$ & $13 \cdot 7$ & $4 \cdot 9$ & $15 \cdot 1$ & $2 \cdot 4$ & $0 \cdot 2$ \\
\hline & \multirow[t]{3}{*}{ Female } & $\begin{array}{l}15-24 \\
25-34 \\
35-44 \\
45-54 \\
55-64 \\
65+\end{array}$ & $\begin{array}{l}170 \\
173 \\
216 \\
224 \\
176 \\
222\end{array}$ & $\begin{array}{r}9 \\
26 \\
67 \\
113 \\
142 \\
215\end{array}$ & $\begin{array}{r}0 \\
0 \\
3 \\
19 \\
61 \\
153\end{array}$ & $\begin{array}{r}0 \\
0 \\
1 \\
4 \\
13 \\
64\end{array}$ & $\begin{array}{r}0 \\
1 \\
8 \\
29 \\
66 \\
131\end{array}$ & $\begin{array}{r}0 \\
0 \\
0 \\
2 \\
6 \\
39\end{array}$ & $\begin{array}{l}0 \\
0 \\
0 \\
0 \\
0 \\
7\end{array}$ & $\begin{array}{r}5 \cdot 3 \\
15 \cdot 0 \\
31 \cdot 0 \\
50 \cdot 5 \\
80 \cdot 7 \\
96 \cdot 8\end{array}$ & $\begin{array}{l}0 \\
0 \\
1 \cdot 4 \\
8 \cdot 5 \\
34 \cdot 7 \\
68 \cdot 9\end{array}$ & $\begin{array}{l}0 \\
0 \\
0 \cdot 5 \\
1 \cdot 8 \\
7 \cdot 4 \\
28 \cdot 8\end{array}$ & $\begin{array}{l}0 \\
0 \cdot 6 \\
3 \cdot 7 \\
13 \cdot 0 \\
37 \cdot 5 \\
59 \cdot 0\end{array}$ & \begin{tabular}{|c|}
0 \\
0 \\
0 \\
$0 \cdot 9$ \\
$3 \cdot 4$ \\
$17 \cdot 6$
\end{tabular} & $\begin{array}{l}0 \\
0 \\
0 \\
0 \\
0 \\
3 \cdot 2\end{array}$ \\
\hline & & Total $15+$ & 1180 & 572 & 236 & 82 & 235 & 47 & 7 & $48 \cdot 6$ & $20 \cdot 0$ & $7 \cdot 0$ & $19 \cdot 9$ & $4 \cdot 0$ & $0 \cdot 6$ \\
\hline & & \multicolumn{8}{|c|}{ Unweighted Mean } & $46 \cdot 5$ & $18 \cdot 9$ & $6 \cdot 4$ & $19 \cdot 0$ & $3 \cdot 7$ & $0 \cdot 5$ \\
\hline \multirow[t]{2}{*}{11} & Male & $\begin{array}{l}35-44 \\
45-54 \\
55-64 \\
65+\end{array}$ & $\begin{array}{l}166 \\
201 \\
127 \\
120\end{array}$ & $\begin{array}{r}65 \\
133 \\
107 \\
115\end{array}$ & $\begin{array}{r}4 \\
28 \\
45 \\
76\end{array}$ & $\begin{array}{r}0 \\
6 \\
14 \\
45\end{array}$ & $\begin{array}{l}10 \\
35 \\
33 \\
68\end{array}$ & $\begin{array}{r}0 \\
0 \\
5 \\
21\end{array}$ & $\begin{array}{l}0 \\
0 \\
0 \\
2\end{array}$ & $\begin{array}{l}49 \cdot 2 \\
65 \cdot 2 \\
84 \cdot 3 \\
95 \cdot 8\end{array}$ & $\begin{array}{r}2 \cdot 4 \\
13 \cdot 9 \\
35 \cdot 4 \\
63 \cdot 3\end{array}$ & $\begin{array}{l}0 \\
3 \cdot 0 \\
11 \cdot 0 \\
37 \cdot 5\end{array}$ & $\begin{array}{r}6 \cdot 0 \\
17 \cdot 4 \\
25 \cdot 9 \\
56 \cdot 7\end{array}$ & $\begin{array}{l}0 \\
0 \\
3 \cdot 9 \\
17 \cdot 5\end{array}$ & $\begin{array}{l}0 \\
0 \\
0 \\
1 \cdot 7\end{array}$ \\
\hline & Female & $\begin{array}{l}35-44 \\
45-54 \\
55-64 \\
65+\end{array}$ & $\begin{array}{l}185 \\
204 \\
160 \\
163\end{array}$ & $\begin{array}{r}69 \\
129 \\
138 \\
162\end{array}$ & $\begin{array}{r}3 \\
27 \\
77 \\
128\end{array}$ & $\begin{array}{r}2 \\
5 \\
25 \\
77\end{array}$ & $\begin{array}{r}11 \\
33 \\
68 \\
113\end{array}$ & $\begin{array}{r}0 \\
3 \\
9 \\
38\end{array}$ & $\begin{array}{r}0 \\
1 \\
1 \\
13\end{array}$ & $\begin{array}{l}37 \cdot 3 \\
63 \cdot 2 \\
86 \cdot 3 \\
99 \cdot 4\end{array}$ & $\begin{array}{r}1 \cdot 6 \\
13 \cdot 2 \\
48 \cdot 2 \\
78 \cdot 5\end{array}$ & $\begin{array}{r}1 \cdot 1 \\
2 \cdot 5 \\
15 \cdot 6 \\
47 \cdot 2\end{array}$ & $\begin{array}{r}6 \cdot 0 \\
16 \cdot 2 \\
42 \cdot 5 \\
69 \cdot 3\end{array}$ & $\begin{array}{l}0 \\
1 \cdot 5 \\
5 \cdot 6 \\
23 \cdot 3\end{array}$ & 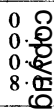 \\
\hline \multirow[t]{2}{*}{13} & Male & $\begin{array}{l}55-64 \\
65+\end{array}$ & $\begin{array}{l}125 \\
120\end{array}$ & $\begin{array}{l}107 \\
116\end{array}$ & $\begin{array}{l}51 \\
80\end{array}$ & $\begin{array}{l}16 \\
51\end{array}$ & $\begin{array}{l}34 \\
76\end{array}$ & $\begin{array}{r}5 \\
23\end{array}$ & $\begin{array}{l}0 \\
2\end{array}$ & $\begin{array}{l}85 \cdot 6 \\
96 \cdot 7\end{array}$ & $\begin{array}{l}40 \cdot 8 \\
66 \cdot 7\end{array}$ & $\begin{array}{l}12 \cdot 8 \\
42 \cdot 5\end{array}$ & $\begin{array}{l}37 \cdot 2 \\
63 \cdot 3\end{array}$ & $\begin{array}{r}4 \cdot 0 \\
19 \cdot 2\end{array}$ & $0 \stackrel{7}{7}$ \\
\hline & Female & $\begin{array}{l}55-64 \\
65+\end{array}$ & $\begin{array}{l}154 \\
160\end{array}$ & $\begin{array}{l}134 \\
159\end{array}$ & $\begin{array}{r}79 \\
130\end{array}$ & $\begin{array}{l}28 \\
80\end{array}$ & $\begin{array}{r}67 \\
114\end{array}$ & $\begin{array}{r}8 \\
41\end{array}$ & $\begin{array}{r}1 \\
13\end{array}$ & $\begin{array}{l}87 \cdot 0 \\
99 \cdot 4\end{array}$ & $\begin{array}{l}51 \cdot 3 \\
81 \cdot 2\end{array}$ & $\begin{array}{l}18 \cdot 2 \\
50 \cdot 0\end{array}$ & $\begin{array}{l}43 \cdot 5 \\
71 \cdot 3\end{array}$ & $\begin{array}{r}5 \cdot 2 \\
25 \cdot 6\end{array}$ & $\begin{array}{l}0 \cdot 6 \\
8 \cdot 1\end{array}$ \\
\hline
\end{tabular}

be seen by comparison with the prevalence values weighted by the age distribution in England and Wales in 1961 (weighted mean) which are also given in Table IIA. Table III shows the weights given in this comparison together with those applicable if the age distribution of 1951 had been used. It will be seen that Table IIA shows more agreement between the two sexes in the unweighted than in the weighted means; this is due to the sex differences in age distribution? demonstrated in Table III.

TABLE III

POPULATION OF ENGLAND AND WALES AGED 15+ YEARS BASED ON THE REGISTRAR GENERAL'S DATA (Population Census 1961, General Volume)

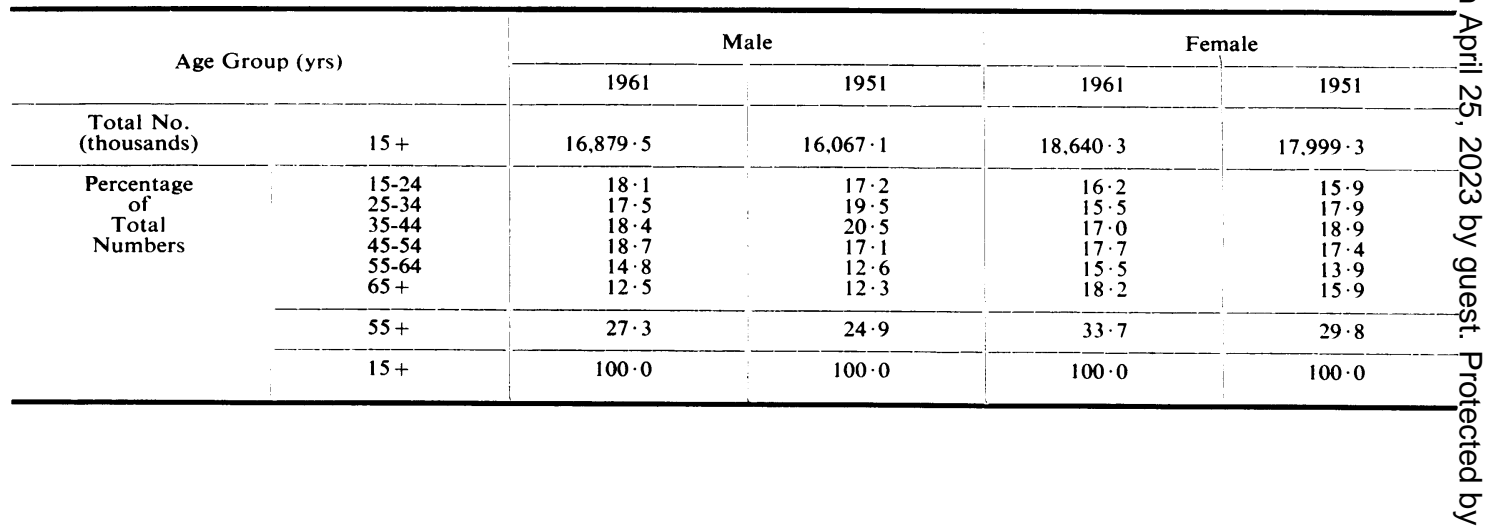


It is suggested that in all surveys in which adequate numbers are available in each age group, the "unweighted mean" prevalence should be given so as to facilitate the comparison between surveys in different countries and at different times.

In the standard population using unweighted means, 52 per cent. of males and 51 per cent. of females had Grade 2 to 4 osteo-arthrosis in one joint or other. Grade 1 osteo-arthrosis has been omitted from Tables IIA and B since this is the doubtful category. There was a female preponderance of both those with $3+$ and $5+$ joint groups involved at the Grade 2 to 4 level. This preponderance did not appear till after the age of 55 . Grade 3 to 4 osteo-arthrosis was present in one joint or other in 19 per cent. of males and 22 per cent. of females. Up to age 55 the prevalence was slightly greater in males and after this in females.

Conclusions as to the number of joints involved are of course influenced by the number of joints $x$ rayed. Since in many population surveys only the hands, feet, and cervical spine are included as a routine, data which would be obtained from such a survey are given in Table IIB under the heading "Nine". Involvement of five or more groups of joints at Grade 2 to 4 osteo-arthrosis was present in only 5 per cent. of males and 6 per cent. of females (unweighted mean) when only these three $x$ rays were used, as compared with 9 and 12 per cent. in Table IIA.

When $x$ rays of the knees and lumbar spine were included giving a coverage of eleven joint groups, the numbers involved closely approached that in the total sample in each of the age groups in which these joints were routinely $x$ rayed. Inclusion of the hips and sacro-iliac joints still further increased the number of joints involved, so that 43 per cent. of males and 50 per cent. of females aged 65 and over had five or more groups of joints affected, compared with 24 per cent. and 29 per cent. in those with $x$ rays of the hands, feet, and neck.

\section{Relationship of Symptoms to $\boldsymbol{X}$ Ray Changes}

Since rheumatoid arthritis (RA) is not an uncommon cause of joint pain, it has been necessary to consider those with and without rheumatoid arthritis separately in studying symptoms. In order to be certain that rheumatoid arthritis was excluded from the non-rheumatoid group, a rather wider definition than usual has been used. Those with probable or definite disease by the American Rheumatism Association's criteria have, of course, been included in the rheumatoid group. Those not falling into this category but considered by the clinician to have definite (Grade 2 to 4 ) rheumatoid arthritis, those with a past history of polyarthritis or with Grade 2 to 4 radiological evidence of the disease or a positive sheep-cell agglutination test (SCAT) were included in an intermediate group. In the present population samples of Leigh and Wensleydale, 26 males and 75 females had probable or definite rheumatoid arthritis, but when this intermediate group was included 200 males and 296 females fell into the "rheumatoid" group. Tables IV to VIII and their accompanying histograms are all arranged in a similar way in order to show clearly the numbers of subjects involved and in part of the Tables the full age grouping. Since many of the subgroups by age, particularly in the higher grades of osteo-arthrosis are extremely small or show nil entries, over-all percentage prevalences of symptoms have been worked out from the total numbers without adjustment to any particular pattern of age distribution. An inspection of the Tables will however support our thesis that age does not appear to affect the relationship of symptoms to osteo-arthrosis but that, in most joint groups examined, symptoms are closely related to the degree of radiological osteoarthrosis. The reader may notice from the Tables that a larger proportion of total rheumatoids than total non-rheumatoids showed definite osteoarthrosis (Grade 2 to 4). This is, however, merely a function of the differing age distribution in the two groups: from the data presented in Tables IV to VI, we worked out the average percentage prevalence of definite osteo-arthrosis in the three groups of hand joints examined for each age group and give below the unweighted mean prevalence for the two broad age groups 15 to 44 years and $45+$ years, "rheumatoid" comprising here both "intermediate" and "probable and definite".

PERCENTAGE PREVALENCE OF GRADE 2 TO 4 OSTEO-ARTHROSIS IN AVERAGE OF THREE JOINT GROUPS, UNWEIGHTED MEANS OF THREE AGE GROUPS

\begin{tabular}{c|c|c|c}
\hline $\begin{array}{c}\text { Age } \\
\text { (yrs) }\end{array}$ & Sex & Non-rheumatoid & Rheumatoid \\
\hline $15-44$ & M & $1 \cdot 3$ & $2 \cdot 1$ \\
& F & $1 \cdot 2$ & $1 \cdot 6$ \\
\hline $45+$ & M & $28 \cdot 4$ & $29 \cdot 9$ \\
& F & $35 \cdot 3$ & $37 \cdot 2$ \\
\hline
\end{tabular}

It will be observed that, when a correction is made for age in this way, the prevalence of osteo-arthrosis was only slightly greater in the rheumatoid group.

In a similar way, i.e. for the average of the three joint groups and as unweighted means, we worked out the percentage of subjects with pain amongst 
those with no or only doubtful osteo-arthrosis (Grade 0 to 1).

PERCENTAGE WITH PAIN IN THE APPROPRIATE DISTRIBUTION, UNWEIGHTED MEANS OF THREE AGE GROUPS

\begin{tabular}{c|c|c|c}
\hline $\begin{array}{c}\text { Age } \\
\text { (yrs) }\end{array}$ & Sex & Non-rheumatoid & Rheumatoid \\
\hline $15-44$ & M & $2 \cdot 4$ & $18 \cdot 4$ \\
\hline $45+$ & F & $3 \cdot 2$ & $19 \cdot 7$ \\
\hline & M & $4 \cdot 1$ & $16 \cdot 4$ \\
& F & $7 \cdot 5$ & $26 \cdot 8$ \\
\hline
\end{tabular}

The slight increase of pain in the older age group may well be accounted for by the greater proportion with doubtful osteo-arthrosis. As the higher grade of osteo-arthrosis are too rare in the younger agE groups, a similar calculation was not possible fot these grades.

The distal interphalangeal joints of the fingers ma be cited as an example. Osteo-arthrosis (Gradet 2 to 4) was present in 246 males and 351 females from a total of 1,097 males and 1,195 females whe had $x$ rays of the hands (Table IV and Fig. 2) opposite).

Of the males who were $x$ rayed, 52 stated that the had pain in the distal interphalangeal joints. Whem the 200 males in the two rheumatoid groups were

TABLE IV

RELATIONSHIP OF SYMPTOMS TO RADIOLOGICAL EVIDENCE OF OSTEOARTHROSIS
IN THE DISTAL INTERPHALANGEAL JOINTS OF THE FINGERS IN THE LEIGH AND WENSLEYDALE ENLARGED SAMPLES (cf. Fig. 2)

\begin{tabular}{|c|c|c|c|c|c|c|c|c|c|c|c|c|}
\hline \multirow{4}{*}{ Sex } & \multirow{4}{*}{ RA or Non-RA } & \multirow{4}{*}{$\begin{array}{c}\text { Age } \\
\text { Group } \\
\text { (yrs) }\end{array}$} & \multirow{4}{*}{$x^{\text {Total }}$ rayed } & \multicolumn{9}{|c|}{ Grade of Osteo-arthrosis (Radiological) } \\
\hline & & & & \multicolumn{3}{|c|}{$0-1$} & \multicolumn{3}{|c|}{2} & \multicolumn{3}{|c|}{$3-4$} \\
\hline & & & & \multirow{2}{*}{$\begin{array}{c}\text { Total } \\
\text { No. }\end{array}$} & \multicolumn{2}{|c|}{ With Pain } & \multirow{2}{*}{$\begin{array}{l}\text { Total } \\
\text { No. }\end{array}$} & \multicolumn{2}{|c|}{ With Pain } & \multirow{2}{*}{$\begin{array}{c}\text { Total } \\
\text { No. }\end{array}$} & \multicolumn{2}{|c|}{ With Pain } \\
\hline & & & & & No. & $\begin{array}{c}\text { Per } \\
\text { cent of } \\
\text { Total }\end{array}$ & & No. & $\begin{array}{c}\text { Per } \\
\text { cent. of } \\
\text { Total }\end{array}$ & & No. & $\begin{array}{l}\text { Per } \overrightarrow{0} \\
\text { cent. of } \\
\text { Togi }\end{array}$ \\
\hline \multirow{6}{*}{ Male } & \multirow[t]{2}{*}{ (a) Non-RA } & $\begin{array}{l}15-24 \\
25-34 \\
35-44 \\
45-54 \\
55-64 \\
65+\end{array}$ & $\begin{array}{r}166 \\
169 \\
165 \\
200 \\
104 \\
93\end{array}$ & $\begin{array}{r}166 \\
166 \\
156 \\
155 \\
55 \\
32\end{array}$ & $\begin{array}{l}0 \\
3 \\
5 \\
1 \\
0 \\
1\end{array}$ & $\begin{array}{l}0 \\
1 \cdot 8 \\
3 \cdot 2 \\
0 \cdot 6 \\
0 \\
3 \cdot 1\end{array}$ & $\begin{array}{r}0 \\
2 \\
8 \\
40 \\
41 \\
44\end{array}$ & $\begin{array}{l}-\overline{0} \\
1 \\
3 \\
5 \\
2\end{array}$ & $\begin{array}{r}\overline{0} \\
12 \cdot 5 \\
7 \cdot 5 \\
12 \cdot 2 \\
4 \cdot 6\end{array}$ & $\begin{array}{r}0 \\
1 \\
1 \\
5 \\
8 \\
17\end{array}$ & $\begin{array}{l}- \\
0 \\
0 \\
0 \\
0 \\
3\end{array}$ & 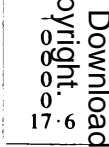 \\
\hline & & Total & 897 & 730 & 10 & $1 \cdot 4$ & 135 & 11 & $8 \cdot 1$ & 32 & 3 & $9 \cdot 4 * \bar{c}$ \\
\hline & (b) RA Intermediate & Total & 174 & 100 & 9 & $9 \cdot 0$ & 55 & 7 & $12 \cdot 7$ & 19 & 5 & $26 \cdot 3=$ \\
\hline & (c) RA Probable and Definite & Total & 26 & 21 & 5 & $23 \cdot 8$ & 3 & 0 & 0 & 2 & 2 & 100 \\
\hline & \multirow[t]{2}{*}{$(b)+(c)$} & $\begin{array}{l}15-24 \\
25-34 \\
35-44 \\
45-54 \\
55-64 \\
65+ \\
\end{array}$ & $\begin{array}{l}14 \\
14 \\
22 \\
39 \\
40 \\
71\end{array}$ & $\begin{array}{l}13 \\
14 \\
22 \\
27 \\
22 \\
23\end{array}$ & $\begin{array}{l}1 \\
1 \\
2 \\
4 \\
4 \\
2\end{array}$ & $\begin{array}{r}7 \cdot 7 \\
7 \cdot 1 \\
9 \cdot 1 \\
14 \cdot 8 \\
18 \cdot 2 \\
8 \cdot 7\end{array}$ & $\begin{array}{r}0 \\
0 \\
0 \\
10 \\
15 \\
33\end{array}$ & $\begin{array}{l}- \\
1 \\
4 \\
2\end{array}$ & $\begin{array}{r}\overline{-} \\
\overline{-} \\
10 \cdot 0 \\
26 \cdot 7 \\
6 \cdot 1\end{array}$ & $\begin{array}{r}1 \\
0 \\
0 \\
2 \\
3 \\
15\end{array}$ & $\begin{array}{l}1 \\
1 \\
1 \\
4\end{array}$ & $\begin{array}{r}100 \\
-? \\
50 \cdot 0 \\
33 \cdot 3 \\
26 \cdot 7\end{array}$ \\
\hline & & Total & 200 & 121 & 14 & $11 \cdot 6$ & 58 & 7 & $12 \cdot 1$ & 21 & 7 & $33 \cdot 3 *=$ \\
\hline \multirow{6}{*}{ Female } & \multirow[t]{2}{*}{ (a) Non-RA } & $\begin{array}{l}15-24 \\
25-34 \\
35-44 \\
45-54 \\
55-64 \\
65+\end{array}$ & $\begin{array}{l}164 \\
157 \\
181 \\
170 \\
115 \\
112\end{array}$ & $\begin{array}{r}164 \\
157 \\
167 \\
131 \\
52 \\
22\end{array}$ & $\begin{array}{l}0 \\
2 \\
1 \\
4 \\
2 \\
0\end{array}$ & $\begin{array}{l}0 \\
1 \cdot 3 \\
0 \cdot 6 \\
3 \cdot 1 \\
3 \cdot 9 \\
0\end{array}$ & $\begin{array}{r}0 \\
0 \\
12 \\
36 \\
49 \\
49\end{array}$ & $\begin{array}{l}- \\
3 \\
4 \\
2\end{array}$ & $\begin{array}{l}- \\
\overline{-} \\
8 \cdot 3 \\
8 \cdot 2 \\
4 \cdot 1\end{array}$ & $\begin{array}{r}0 \\
0 \\
2 \\
3 \\
14 \\
41\end{array}$ & $\begin{array}{l}- \\
1 \\
4 \\
10\end{array}$ & $\begin{array}{r}-\quad \\
-\quad \supseteqq \\
33.3 \\
28.6 \\
24.4\end{array}$ \\
\hline & & Total & 899 & 693 & 9 & $1 \cdot 3$ & 146 & 9 & $6 \cdot 2$ & 60 & 15 & $25 \cdot 0 *$ \\
\hline & (b) RA Intermediate & Total & 221 & 122 & 11 & $9 \cdot 0$ & 66 & 17 & $25 \cdot 8$ & 33 & 15 & $45 \cdot 5 \mathrm{c}$ \\
\hline & (c) RA Probable and Definite & Total & 75 & 29 & 7 & $24 \cdot 1$ & 24 & 7 & $29 \cdot 2$ & 22 & 10 & $45 \cdot 5 \mathrm{~N}$ \\
\hline & \multirow[t]{2}{*}{$(b)+(c)$} & $\begin{array}{l}15-24 \\
25-34 \\
35-44 \\
45-54 \\
55-64 \\
65+\end{array}$ & $\begin{array}{r}8 \\
20 \\
35 \\
58 \\
64 \\
111\end{array}$ & $\begin{array}{r}8 \\
20 \\
34 \\
44 \\
30 \\
15\end{array}$ & $\begin{array}{l}2 \\
0 \\
5 \\
4 \\
7 \\
0\end{array}$ & $\begin{array}{r}25 \cdot 0 \\
0 \cdot 0 \\
14 \cdot 7 \\
9 \cdot 1 \\
23 \cdot 3 \\
0 \cdot 0\end{array}$ & $\begin{array}{l}0 \\
0 \\
1 \\
10 \\
25 \\
54\end{array}$ & $\begin{array}{r}- \\
0 \\
1 \\
6 \\
17\end{array}$ & $\begin{array}{c}- \\
\overline{0} \\
10 \cdot 0 \\
24 \cdot 0 \\
31 \cdot 5\end{array}$ & $\begin{array}{r}0 \\
0 \\
0 \\
4 \\
9 \\
42 \\
\end{array}$ & $\begin{array}{r}- \\
- \\
2 \\
3 \\
20\end{array}$ & 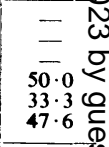 \\
\hline & & Total & 296 & 151 & 18 & $11 \cdot 9$ & 90 & 24 & $26 \cdot 7$ & 55 & 25 & $45 \cdot 5 \%$ \\
\hline
\end{tabular}

Probability of difference in proportion of pain between Osteo-arthrosis Grades $0-1$ and $3-4: \quad *=<0.05 \quad * *=<0.01$ 


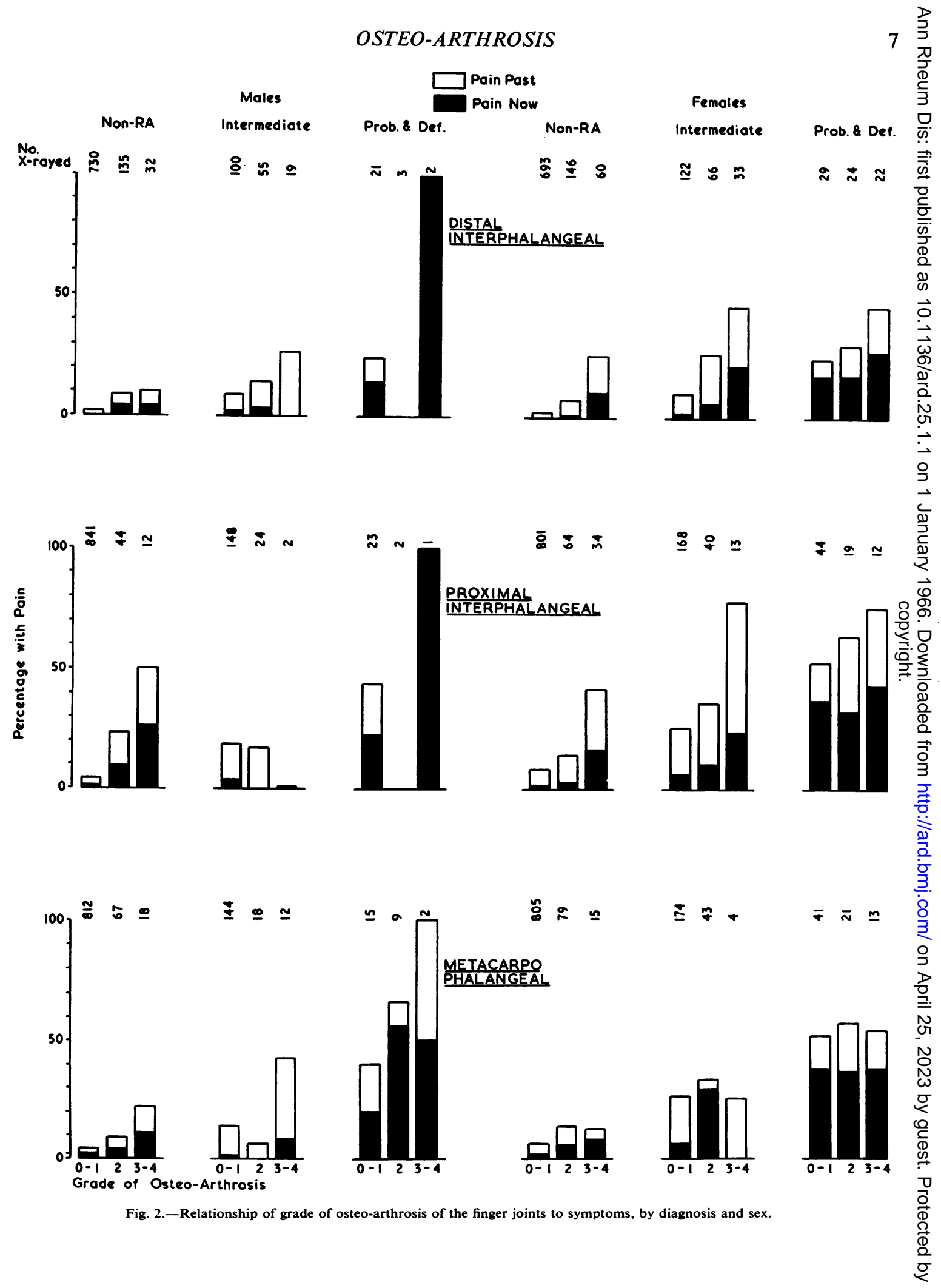


RELATIONSHIP OF SYMPTOMS TO RADIOLOGICAL EVIDENCE OF OSTEO-ARTHROSIS IN THE PROXIMAL INTERPHALANGEAL JOINTS OF THE FINGERS IN THE LEIGH AND WENSLEYDALE ENLARGED SAMPLES (cf. Fig. 2)

\begin{tabular}{|c|c|c|c|c|c|c|c|c|c|c|c|c|}
\hline \multirow{4}{*}{ Sex } & \multirow{4}{*}{ RA or Non-RA } & \multirow{4}{*}{$\begin{array}{c}\text { Age } \\
\text { Group } \\
\text { (yrs) }\end{array}$} & \multirow{4}{*}{$\underset{x \text { rayed }}{\text { Total }}$} & \multicolumn{9}{|c|}{ Grade of Osteo-arthrosis (Radiological) } \\
\hline & & & & \multicolumn{3}{|c|}{$0-1$} & \multicolumn{3}{|c|}{2} & \multicolumn{3}{|c|}{$3-4$} \\
\hline & & & & \multirow{2}{*}{$\begin{array}{c}\text { Total } \\
\text { No. }\end{array}$} & \multicolumn{2}{|c|}{ With Pain } & \multirow{2}{*}{$\begin{array}{c}\text { Total } \\
\text { No. }\end{array}$} & \multicolumn{2}{|c|}{ With Pain } & \multirow{2}{*}{$\begin{array}{l}\text { Total } \\
\text { No. }\end{array}$} & \multicolumn{2}{|c|}{ With Pain } \\
\hline & & & & & No. & $\begin{array}{c}\text { Per } \\
\text { cent. of } \\
\text { Total }\end{array}$ & & No. & $\begin{array}{l}\text { Per } \\
\text { cent. of } \\
\text { Total }\end{array}$ & & No. & $\begin{array}{l}\text { Per } \\
\text { cent. of } \\
\text { Total }\end{array}$ \\
\hline \multirow{6}{*}{ Male } & \multirow[t]{2}{*}{ (a) Non-RA } & $\begin{array}{l}15-24 \\
25-34 \\
35-44 \\
45-54 \\
55-64 \\
65+\end{array}$ & $\begin{array}{r}166 \\
169 \\
165 \\
200 \\
104 \\
93\end{array}$ & $\begin{array}{r}166 \\
169 \\
161 \\
189 \\
93 \\
63\end{array}$ & $\begin{array}{r}2 \\
8 \\
5 \\
15 \\
3 \\
3\end{array}$ & $\begin{array}{l}1 \cdot 2 \\
4 \cdot 7 \\
3 \cdot 1 \\
7 \cdot 9 \\
3 \cdot 2 \\
4 \cdot 8\end{array}$ & $\begin{array}{r}0 \\
0 \\
4 \\
11 \\
10 \\
19\end{array}$ & $\begin{array}{c}- \\
1 \\
4 \\
2 \\
3\end{array}$ & $\begin{array}{c}- \\
\overline{25 \cdot 0} \\
36 \cdot 4 \\
20 \cdot 0 \\
15 \cdot 8\end{array}$ & $\begin{array}{r}0 \\
0 \\
0 \\
0 \\
1 \\
11\end{array}$ & $\frac{-}{-}$ & $\begin{array}{l}=\frac{0}{0} \\
=\bar{c} \\
54.5\end{array}$ \\
\hline & & Total & 897 & 841 & 36 & $4 \cdot 3$ & 44 & 10 & $22 \cdot 7$ & 12 & 6 & $50 \cdot 0$ \\
\hline & (b) RA Intermediate & Total & 174 & 148 & 27 & $18 \cdot 2$ & 24 & 4 & $16 \cdot 7$ & 2 & 0 & 0 \\
\hline & (c) RA Probable and Definite & Total & 26 & 23 & 10 & $43 \cdot 5$ & 2 & 0 & 0 & 1 & 1 & $100 \cdot 0 \mathrm{c}$ \\
\hline & \multirow[t]{2}{*}{$(b)+(c)$} & $\begin{array}{l}15-24 \\
25-34 \\
35-44 \\
45-54 \\
55-64 \\
65+\end{array}$ & $\begin{array}{l}14 \\
14 \\
22 \\
39 \\
40 \\
71\end{array}$ & $\begin{array}{l}14 \\
14 \\
22 \\
35 \\
36 \\
50\end{array}$ & $\begin{array}{r}2 \\
4 \\
6 \\
6 \\
9 \\
10\end{array}$ & $\begin{array}{l}14 \cdot 3 \\
28 \cdot 6 \\
27 \cdot 3 \\
17 \cdot 1 \\
25 \cdot 0 \\
20 \cdot 0\end{array}$ & $\begin{array}{r}0 \\
0 \\
0 \\
4 \\
4 \\
18\end{array}$ & $\begin{array}{l}- \\
- \\
1 \\
1 \\
2\end{array}$ & $\begin{array}{l}- \\
\bar{Z} \\
25 \cdot 0 \\
25 \cdot 0 \\
11 \cdot 1\end{array}$ & $\begin{array}{l}0 \\
0 \\
0 \\
0 \\
0 \\
3\end{array}$ & $\bar{E} \frac{}{-}$ & $\begin{array}{l}= \\
= \\
\overline{33 \cdot 36}\end{array}$ \\
\hline & & Total & 200 & 171 & 37 & $21 \cdot 6$ & 26 & 4 & $15 \cdot 4$ & 3 & 1 & $33 \cdot 3 \bar{c}$ \\
\hline \multirow{6}{*}{ Female } & \multirow[t]{2}{*}{ (a) Non-RA } & $\begin{array}{l}15-24 \\
25-34 \\
35-44 \\
45-54 \\
55-64 \\
65+\end{array}$ & $\begin{array}{l}164 \\
157 \\
181 \\
170 \\
115 \\
112\end{array}$ & $\begin{array}{r}164 \\
157 \\
181 \\
160 \\
82 \\
57\end{array}$ & $\begin{array}{r}2 \\
8 \\
16 \\
14 \\
9 \\
5\end{array}$ & $\begin{array}{r}1 \cdot 2 \\
5 \cdot 1 \\
8 \cdot 8 \\
8 \cdot 8 \\
11 \cdot 0 \\
8 \cdot 8\end{array}$ & $\begin{array}{r}0 \\
0 \\
0 \\
8 \\
25 \\
31\end{array}$ & $\begin{array}{c}- \\
- \\
2 \\
2 \\
5\end{array}$ & $\begin{array}{r}- \\
\bar{Z} \\
25 \cdot 0 \\
8 \cdot 0 \\
16 \cdot 1\end{array}$ & $\begin{array}{r}0 \\
0 \\
0 \\
2 \\
8 \\
24\end{array}$ & $\begin{array}{c}- \\
\overline{1} \\
4 \\
9\end{array}$ & - \\
\hline & & Total & 899 & 801 & 54 & $6 \cdot 8$ & 64 & 9 & $14 \cdot 1$ & 34 & 14 & $4 \cos ^{2}$ \\
\hline & (b) RA Intermediate & Total & 221 & 168 & 44 & $26 \cdot 2$ & 40 & 14 & $35 \cdot 0$ & 13 & 10 & 76 \\
\hline & (c) RA Probable and Definite & Total & 75 & 44 & 23 & $52 \cdot 3$ & 19 & 12 & $63 \cdot 2$ & 12 & 9 & $75 \cdot 0$ 8 \\
\hline & \multirow[t]{2}{*}{$(b)+(c)$} & $\begin{array}{l}15-24 \\
25-34 \\
35-44 \\
45-54 \\
55-64 \\
65+\end{array}$ & $\begin{array}{r}8 \\
20 \\
35 \\
58 \\
64 \\
111 \\
\end{array}$ & $\begin{array}{r}8 \\
20 \\
34 \\
53 \\
45 \\
52 \\
\end{array}$ & $\begin{array}{r}2 \\
4 \\
7 \\
21 \\
16 \\
17 \\
\end{array}$ & $\begin{array}{l}25.0 \\
20.0 \\
20.6 \\
39.6 \\
35.6 \\
32.7\end{array}$ & $\begin{array}{r}0 \\
0 \\
1 \\
4 \\
16 \\
38 \\
\end{array}$ & $\begin{array}{r}- \\
1 \\
1 \\
6 \\
18\end{array}$ & $\begin{array}{r}- \\
100 \cdot 0 \\
25 \cdot 0 \\
37 \cdot 5 \\
47 \cdot 4\end{array}$ & $\begin{array}{r}0 \\
0 \\
0 \\
1 \\
3 \\
21\end{array}$ & $\begin{array}{c}- \\
\overline{1} \\
2 \\
16\end{array}$ & 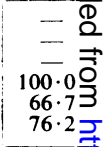 \\
\hline & & Total & 296 & 212 & 67 & $31 \cdot 6$ & 59 & 26 & $44 \cdot 1$ & 25 & 19 & $76 \cdot 0$ \\
\hline
\end{tabular}

Probability of difference in proportion of pain between Osteo-arthrosis Grades 0-1 and 3-4: $*=<0 \cdot 05 * *=<0.01$.

excluded, only 24 males had pain, the proportion rising from 1 per cent. in those without definite radiological evidence of osteo-arthrosis to 9 per cent. in those with Grade 3 to 4 change. Of the females, 100 stated that they had had pain in the distal interphalangeal joints but of the 899 non-rheumatoid females only 33 had had pain, the prevalence rising from 1 per cent. in those without definite evidence of osteo-arthrosis to 25 per cent. in those with Grade 3 to 4 change.

Of the 174 males in the "intermediate group", 21 had had pain in the distal interphalangeal joints, the prevalence rising from 9 per cent. in those with Grade 0 to 1 osteo-arthrosis to 26 per cent. in those with Grade 3 to 4 change. In the 221 females in the intermediate group it rose from 9 to 46 per cent.

There were only 26 males with probable of definite rheumatoid arthritis and seven of these has had pain in the distal interphalangeal joints, five $\vec{p}$ the 21 with Grade 0 to 1 osteo-arthrosis and all those with Grade 3 to 4 change. Of the 75 females with probable or definite disease, 24 had had pain in thle distal interphalangeal joints, the frequency varying from 24 to 46 per cent. with increasing severity of osteo-arthrosis.

The general pattern is the same in all the peripher的 joints. There is a gradient in the frequency of sympe toms between those with Grade 0 to 1 and 3 to $\bar{d}$ 
TABLE VI

RELATIONSHIP OF SYMPTOMS TO RADIOLOGICAL EVIDENCE OF OSTEO-ARTHROSIS

IN THE METACARPOPHALANGEAL JOINTS IN THE LEIGH AND WENSLEYDALE ENLARGED SAMPLES (cf. Fig. 2)

\begin{tabular}{|c|c|c|c|c|c|c|c|c|c|c|c|c|}
\hline \multirow{4}{*}{ Sex } & \multirow{4}{*}{ RA or Non-RA } & \multirow{4}{*}{$\begin{array}{l}\text { Age } \\
\text { Group } \\
\text { (yrs) }\end{array}$} & \multirow{4}{*}{$\underset{x \text { rayed }}{\text { Total }}$} & \multicolumn{9}{|c|}{ Grade of Osteo-arthrosis (Radiological) } \\
\hline & & & & \multicolumn{3}{|c|}{$0-1$} & \multicolumn{3}{|c|}{2} & \multicolumn{3}{|c|}{$3-4$} \\
\hline & & & & \multirow{2}{*}{$\begin{array}{c}\text { Total } \\
\text { No. }\end{array}$} & \multicolumn{2}{|c|}{ With Pain } & \multirow{2}{*}{$\begin{array}{c}\text { Total } \\
\text { No. }\end{array}$} & \multicolumn{2}{|c|}{ With Pain } & \multirow{2}{*}{$\begin{array}{c}\text { Total } \\
\text { No. }\end{array}$} & \multicolumn{2}{|c|}{ With Pain } \\
\hline & & & & & No. & $\begin{array}{c}\text { Per } \\
\text { cent. of } \\
\text { Total }\end{array}$ & & No. & $\begin{array}{c}\text { Per } \\
\text { cent. of } \\
\text { Total }\end{array}$ & & No. & $\begin{array}{c}\text { Per } \\
\text { cent. of } \\
\text { Total }\end{array}$ \\
\hline \multirow{6}{*}{ Male } & \multirow[t]{2}{*}{ (a) Non-RA } & $\begin{array}{l}15-24 \\
25-34 \\
35-44 \\
45-54 \\
55-64 \\
65+\end{array}$ & $\begin{array}{r}166 \\
169 \\
165 \\
200 \\
104 \\
93\end{array}$ & $\begin{array}{r}166 \\
166 \\
165 \\
176 \\
86 \\
53\end{array}$ & $\begin{array}{l}0 \\
7 \\
5 \\
7 \\
5 \\
4\end{array}$ & $\begin{array}{l}0 \\
4 \cdot 2 \\
3 \cdot 0 \\
4 \cdot 0 \\
5 \cdot 8 \\
7 \cdot 6\end{array}$ & $\begin{array}{r}0 \\
2 \\
0 \\
22 \\
15 \\
28\end{array}$ & $\begin{array}{l}-1 \\
2 \\
1 \\
2\end{array}$ & $\begin{array}{l}\overline{50 \cdot 0} \\
\overline{9 \cdot 1} \\
6 \cdot 7 \\
7 \cdot 1\end{array}$ & $\begin{array}{r}0 \\
1 \\
0 \\
2 \\
3 \\
12\end{array}$ & $\begin{array}{l}\overline{0} \\
1 \\
1 \\
2\end{array}$ & $\begin{array}{l}\overline{0} \\
50 \cdot 0 \\
33 \cdot 3 \\
16 \cdot 7\end{array}$ \\
\hline & & Total & 897 & 812 & 28 & $3 \cdot 4$ & 67 & 6 & $9 \cdot 0$ & 18 & 4 & $22 \cdot 2^{*}$ \\
\hline & (b) RA Intermediate & Total & 174 & 144 & 20 & $13 \cdot 9$ & 18 & 1 & $5 \cdot 5$ & 12 & 5 & $41 \cdot 5$ \\
\hline & (c) RA Probable and Definite & Total & 26 & 15 & 6 & $40 \cdot 0$ & 9 & 6 & $66 \cdot 7$ & 2 & 2 & $100 \cdot 0$ \\
\hline & \multirow[t]{2}{*}{$(b)+(c)$} & $\begin{array}{l}15-24 \\
25-34 \\
35-44 \\
45-54 \\
55-64 \\
65+\end{array}$ & $\begin{array}{l}14 \\
14 \\
22 \\
39 \\
40 \\
71\end{array}$ & $\begin{array}{l}14 \\
13 \\
21 \\
33 \\
30 \\
48\end{array}$ & $\begin{array}{l}3 \\
4 \\
4 \\
5 \\
6 \\
4\end{array}$ & $\begin{array}{r}21 \cdot 4 \\
30 \cdot 8 \\
19 \cdot 0 \\
15 \cdot 2 \\
20 \cdot 0 \\
8 \cdot 3\end{array}$ & $\begin{array}{r}0 \\
0 \\
1 \\
6 \\
9 \\
11\end{array}$ & $\begin{array}{l}-\mathbf{0} \\
0 \\
2 \\
2 \\
3\end{array}$ & $\begin{array}{c}\overrightarrow{0} \\
0 \\
33 \cdot 3 \\
22 \cdot 2 \\
27 \cdot 3\end{array}$ & $\begin{array}{l}0 \\
1 \\
0 \\
0 \\
1 \\
12\end{array}$ & $\begin{array}{c}\overline{0} \\
\overline{1} \\
6\end{array}$ & $\begin{array}{c}\overline{0} \\
\overline{-} \\
100 \cdot 0 \\
50 \cdot 0\end{array}$ \\
\hline & & Total & 200 & 159 & 26 & $16 \cdot 4$ & 27 & 7 & $25 \cdot 9$ & 14 & 7 & $50 \cdot 0^{*}$ \\
\hline \multirow{6}{*}{ Female } & \multirow[t]{2}{*}{ (a) Non-RA } & $\begin{array}{l}15-24 \\
25-34 \\
35-44 \\
45-54 \\
55-64 \\
65+\end{array}$ & $\begin{array}{l}164 \\
157 \\
181 \\
170 \\
115 \\
112\end{array}$ & $\begin{array}{r}164 \\
156 \\
177 \\
159 \\
86 \\
63\end{array}$ & $\begin{array}{r}3 \\
7 \\
9 \\
11 \\
9 \\
9\end{array}$ & $\begin{array}{r}1 \cdot 8 \\
4 \cdot 5 \\
5 \cdot 1 \\
6 \cdot 9 \\
10 \cdot 6 \\
14 \cdot 3\end{array}$ & $\begin{array}{r}0 \\
1 \\
3 \\
11 \\
24 \\
40\end{array}$ & $\begin{array}{l}-0 \\
0 \\
0 \\
5 \\
6\end{array}$ & $\begin{array}{c}\overline{0} \\
0 \\
0 \\
20 \cdot 8 \\
15 \cdot 0\end{array}$ & $\begin{array}{l}0 \\
0 \\
1 \\
0 \\
5 \\
9\end{array}$ & $\begin{array}{c}\overline{-} \\
\overline{0} \\
0 \\
2\end{array}$ & $\begin{array}{l}\bar{Z} \\
\overline{0} \\
0 \\
22 \cdot 2\end{array}$ \\
\hline & & Total & 899 & 805 & 48 & $6 \cdot 0$ & 79 & 11 & $13 \cdot 9$ & 15 & 2 & $13 \cdot 3$ \\
\hline & (b) RA Intermediate & Total & 221 & 174 & 45 & $25 \cdot 9$ & 43 & 14 & $32 \cdot 6$ & 4 & 1 & $25 \cdot 0$ \\
\hline & (c) RA Probable and Definite & Total & 75 & 41 & 21 & $51 \cdot 2$ & 21 & 12 & $57 \cdot 2$ & 13 & 7 & $53 \cdot 9$ \\
\hline & \multirow[t]{2}{*}{$(b)+(c)$} & $\begin{array}{l}15-24 \\
25-34 \\
35-44 \\
45-54 \\
55-64 \\
65+\end{array}$ & $\begin{array}{r}8 \\
20 \\
35 \\
58 \\
64 \\
111\end{array}$ & $\begin{array}{r}8 \\
20 \\
32 \\
53 \\
52 \\
50\end{array}$ & $\begin{array}{r}2 \\
5 \\
7 \\
18 \\
17 \\
17\end{array}$ & $\begin{array}{l}25 \cdot 0 \\
25 \cdot 0 \\
21 \cdot 9 \\
34 \cdot 0 \\
32 \cdot 7 \\
34 \cdot 0\end{array}$ & $\begin{array}{r}0 \\
0 \\
2 \\
4 \\
11 \\
47\end{array}$ & $\begin{array}{r}- \\
0 \\
1 \\
6 \\
19\end{array}$ & $\begin{array}{c}\text { 二 } \\
\overline{0} \\
25 \cdot 0 \\
54 \cdot 6 \\
40 \cdot 4\end{array}$ & $\begin{array}{r}0 \\
0 \\
1 \\
1 \\
1 \\
14\end{array}$ & $\begin{array}{l}- \\
1 \\
1 \\
1 \\
5\end{array}$ & $\begin{array}{r}\overline{-} \\
100 \cdot 0 \\
100 \cdot 0 \\
100 \cdot 0 \\
35 \cdot 7\end{array}$ \\
\hline & & Total & 296 & 215 & 66 & $30 \cdot 7$ & 64 & 26 & $40 \cdot 5$ & 17 & 8 & $47 \cdot 1$ \\
\hline
\end{tabular}

Probability of difference in proportion between Osteo-arthrosis Grades 0-1 and 3-4: $*=<0.05 * *=<0 \cdot 01$.

osteo-arthrosis, and this gradient is similar in the non-rheumatoid, intermediate rheumatoid, and rheumatoid groups, though starting on a higher level in the intermediate group and on a still higher level in the probable-definite group. On the whole the gradient is rather greater in those without rheumatoid arthritis. Pain present at the time of the survey shows much the same gradient as total pain.

Exceptions to these generalizations are the wrists and metacarpophalangeal joints. These show little or no relationship between osteo-arthrosis and symptoms in females and the possibility that unrecognized rheumatoid arthritis is obscuring the relationship between osteo-arthrosis and symptoms must be considered. It would seem that rheumatoid arthritis is the main cause of symptoms in these joints in this sex.

The numbers with probable or definite rheumatoid arthritis are small in the males and this accounts for the uneven results.

In view of the difficulty some respondents had in localizing pain in the individual joints of the fingers, a grading was given for generalized osteo-arthrosis in the hand $x$ rays, according to readings made by Prof. J. H. Kellgren. This grading showed a significant association with pain in the hands in the non- 
rheumatoid group and some association in the In the spine the association between osteo intermediate group (Table VII and Fig. 3, opposite). arthrosis of the apophyseal joints and symptoms

TABLE VII

RELATIONSHIP OF SYMPTOMS TO RADIOLOGICAL EVIDENCE OF OSTEO-ARTHROSIS

IN THE LEIGH AND WENSLEYDALE ENLARGED SAMPLES (Fig. 3 Hands, Fig. 4 Spine, Fig. 5 Legs and Feet)

\begin{tabular}{|c|c|c|c|c|c|c|c|c|c|c|c|c|}
\hline \multirow{4}{*}{$\begin{array}{l}\text { Joint } \\
\text { Group }\end{array}$} & \multirow{4}{*}{ Sex } & \multirow{4}{*}{ RA or Non-RA } & \multirow{4}{*}{$\begin{array}{c}\text { Tota! } \\
x \text { rayed }\end{array}$} & \multicolumn{9}{|c|}{ Grade of Osteo-arthrosis (Radiological) } \\
\hline & & & & \multicolumn{3}{|c|}{$0-1$} & \multicolumn{3}{|c|}{2} & \multicolumn{3}{|c|}{$3-4$} \\
\hline & & & & \multirow{2}{*}{$\begin{array}{c}\text { Total } \\
\text { No. }\end{array}$} & \multicolumn{2}{|c|}{ With Pain } & \multirow{2}{*}{$\begin{array}{c}\text { Total } \\
\text { No. }\end{array}$} & \multicolumn{2}{|c|}{ With Pain } & \multirow{2}{*}{$\begin{array}{c}\text { Total } \\
\text { No. }\end{array}$} & \multicolumn{2}{|c|}{ With Pain } \\
\hline & & & & & No. & $\begin{array}{l}\text { Per cent. } \\
\text { of } \\
\text { Total }\end{array}$ & & No. & $\begin{array}{l}\text { Per cent. } \\
\text { of } \\
\text { Total }\end{array}$ & & No. & $\begin{array}{c}\text { Per cent } \\
\text { of } \\
\text { Total }\end{array}$ \\
\hline \multirow[t]{2}{*}{$\begin{array}{l}\text { Carpo- } \\
\text { metacarpal }\end{array}$} & Male & $\begin{array}{l}\text { Non-RA } \\
\text { RA Intermediate } \\
\text { RA Probable and Definite }\end{array}$ & $\begin{array}{r}897 \\
174 \\
26\end{array}$ & $\begin{array}{r}827 \\
143 \\
22\end{array}$ & $\begin{array}{r}18 \\
9 \\
6\end{array}$ & $\begin{array}{r}2 \cdot 2 \\
6 \cdot 3 \\
27 \cdot 3\end{array}$ & $\begin{array}{r}52 \\
21 \\
2\end{array}$ & $\begin{array}{l}2 \\
2 \\
0\end{array}$ & $\begin{array}{l}3 \cdot 8 \\
9 \cdot 5 \\
0 \cdot 0\end{array}$ & $\begin{array}{r}18 \\
10 \\
2\end{array}$ & $\begin{array}{l}6 \\
3 \\
1\end{array}$ & $\left.\begin{array}{l}33 \cdot 3 \\
30 \cdot 0 \\
50 \cdot 0\end{array}\right\}$ \\
\hline & Female & $\begin{array}{l}\text { Non-RA } \\
\text { RA Intermediate } \\
\text { RA Probable and Definite }\end{array}$ & $\begin{array}{r}898 \\
221 \\
75\end{array}$ & $\begin{array}{r}792 \\
167 \\
48\end{array}$ & $\begin{array}{l}27 \\
33 \\
17\end{array}$ & $\begin{array}{r}3 \cdot 4 \\
19 \cdot 8 \\
35 \cdot 4\end{array}$ & $\begin{array}{l}74 \\
36 \\
19\end{array}$ & $\begin{array}{r}15 \\
9 \\
5\end{array}$ & $\begin{array}{l}20 \cdot 2 \\
25 \cdot 0 \\
26 \cdot 3\end{array}$ & $\begin{array}{r}32 \\
18 \\
8\end{array}$ & $\begin{array}{r}11 \\
9 \\
3\end{array}$ & $\left.\begin{array}{l}34 \cdot 4 \\
50 \cdot 0 \\
37 \cdot 5\end{array}\right\}$ \\
\hline \multirow[t]{2}{*}{ Wrists } & Male & $\begin{array}{l}\text { Non-RA } \\
\text { RA Intermediate } \\
\text { RA Probable and Definite }\end{array}$ & $\begin{array}{r}896 \\
172 \\
26\end{array}$ & $\begin{array}{r}838 \\
150 \\
21\end{array}$ & $\begin{array}{r}36 \\
20 \\
7\end{array}$ & $\begin{array}{r}4 \cdot 3 \\
13 \cdot 3 \\
33 \cdot 3\end{array}$ & $\begin{array}{r}44 \\
17 \\
2\end{array}$ & $\begin{array}{l}4 \\
3 \\
1\end{array}$ & $\begin{array}{r}9 \cdot 1 \\
17 \cdot 6 \\
50 \cdot 0\end{array}$ & $\begin{array}{r}14 \\
5 \\
3\end{array}$ & $\begin{array}{l}6 \\
2 \\
3\end{array}$ & $\left.\begin{array}{r}42 \cdot 9 \\
40 \cdot 0 \\
100 \cdot 0\end{array}\right\}$ \\
\hline & Female & $\begin{array}{l}\text { Non-RA } \\
\text { RA Intermediate } \\
\text { RA Probable and Definite }\end{array}$ & $\begin{array}{r}897 \\
219 \\
74\end{array}$ & $\begin{array}{r}871 \\
196 \\
65\end{array}$ & $\begin{array}{l}57 \\
46 \\
19\end{array}$ & $\begin{array}{r}6 \cdot 5 \\
23 \cdot 5 \\
29 \cdot 2\end{array}$ & $\begin{array}{r}24 \\
18 \\
6\end{array}$ & $\begin{array}{l}1 \\
3 \\
3\end{array}$ & $\begin{array}{r}4 \cdot 2 \\
16 \cdot 7 \\
50 \cdot 0\end{array}$ & $\begin{array}{l}2 \\
5 \\
3\end{array}$ & $\begin{array}{l}0 \\
0 \\
1\end{array}$ & $\begin{array}{r}0 \cdot 0 \\
0 \cdot 0 \\
33 \cdot 3\end{array}$ \\
\hline \multirow[t]{2}{*}{$\begin{array}{l}\text { Hands- } \\
\text { Generalized } \\
\text { Osteo- } \\
\text { arthrosis }\end{array}$} & Male & $\begin{array}{l}\text { Non-RA } \\
\text { RA Intermediate } \\
\text { RA Probable and Definite }\end{array}$ & $\begin{array}{r}897 \\
174 \\
26\end{array}$ & $\begin{array}{r}734 \\
95 \\
19\end{array}$ & $\begin{array}{l}19 \\
12 \\
11\end{array}$ & $\begin{array}{r}2 \cdot 6 \\
12 \cdot 6 \\
57 \cdot 9\end{array}$ & $\begin{array}{r}103 \\
41 \\
3\end{array}$ & $\begin{array}{l}7 \\
7 \\
1\end{array}$ & $\begin{array}{r}6 \cdot 8 \\
17 \cdot 1 \\
33 \cdot 3\end{array}$ & $\begin{array}{r}60 \\
38 \\
4\end{array}$ & $\begin{array}{r}11 \\
7 \\
2\end{array}$ & $\begin{array}{l}18 \cdot 3 \\
18 \cdot 8 \\
50 \cdot 8 \\
\end{array}$ \\
\hline & Female & $\begin{array}{l}\text { Non-RA } \\
\text { RA Intermediate } \\
\text { RA Probable and Definite }\end{array}$ & $\begin{array}{r}899 \\
221 \\
75\end{array}$ & $\begin{array}{r}680 \\
112 \\
24\end{array}$ & $\begin{array}{l}29 \\
30 \\
14\end{array}$ & $\begin{array}{r}4 \cdot 3 \\
26 \cdot 8 \\
58 \cdot 3\end{array}$ & $\begin{array}{r}109 \\
47 \\
20\end{array}$ & $\begin{array}{l}23 \\
10 \\
11\end{array}$ & $\begin{array}{l}21 \cdot 1 \\
21 \cdot 3 \\
55 \cdot 0\end{array}$ & $\begin{array}{r}110 \\
62 \\
31\end{array}$ & $\begin{array}{l}30 \\
27 \\
15\end{array}$ & $\begin{array}{l}27 \cdot \frac{5}{4} . \\
43 \cdot \frac{2}{4}\end{array}$ \\
\hline \multirow[t]{2}{*}{$\begin{array}{l}\text { Cervical } \\
\text { Spine }\end{array}$} & Male & $\begin{array}{l}\text { Non-RA } \\
\text { RA Intermediate } \\
\text { RA Probable and Definite }\end{array}$ & $\begin{array}{r}895 \\
173 \\
26\end{array}$ & $\begin{array}{r}812 \\
144 \\
21\end{array}$ & $\begin{array}{r}302 \\
75 \\
9\end{array}$ & $\begin{array}{l}37 \cdot 1 \\
52 \cdot 1 \\
42 \cdot 9\end{array}$ & $\begin{array}{r}67 \\
22 \\
3\end{array}$ & $\begin{array}{r}26 \\
10 \\
2\end{array}$ & $\begin{array}{l}38 \cdot 8 \\
45 \cdot 5 \\
66 \cdot 7\end{array}$ & $\begin{array}{r}16 \\
7 \\
2\end{array}$ & $\begin{array}{r}11 \\
2 \\
2\end{array}$ & $\begin{array}{c}68 \cdot 7^{*} \\
28 \cdot 6 \\
100 \cdot 0\end{array}$ \\
\hline & Female & $\begin{array}{l}\text { Non-RA } \\
\text { RA Intermediate } \\
\text { RA Probable and Definite }\end{array}$ & $\begin{array}{r}892 \\
222 \\
75\end{array}$ & $\begin{array}{r}825 \\
188 \\
57\end{array}$ & $\begin{array}{r}326 \\
122 \\
33\end{array}$ & $\begin{array}{l}39 \cdot 6 \\
64 \cdot 4 \\
57 \cdot 9\end{array}$ & $\begin{array}{l}58 \\
22 \\
14\end{array}$ & $\begin{array}{l}23 \\
18 \\
12\end{array}$ & $\begin{array}{l}39 \cdot 7 \\
81 \cdot 8 \\
85 \cdot 7\end{array}$ & $\begin{array}{r}9 \\
12 \\
4\end{array}$ & $\begin{array}{l}5 \\
8 \\
3\end{array}$ & $\begin{array}{l}55 \cdot 5 \\
66 \cdot 7 \\
75 \cdot 0\end{array}$ \\
\hline \multirow[t]{2}{*}{$\begin{array}{l}\text { Lumbar } \\
\text { Spine }\end{array}$} & Male & $\begin{array}{l}\text { Non-RA } \\
\text { RA Intermediate } \\
\text { RA Probable and Definite }\end{array}$ & $\begin{array}{r}483 \\
125 \\
22\end{array}$ & $\begin{array}{r}385 \\
96 \\
14\end{array}$ & $\begin{array}{r}199 \\
42 \\
4\end{array}$ & $\begin{array}{l}51 \cdot 7 \\
43 \cdot 8 \\
28 \cdot 6\end{array}$ & $\begin{array}{r}78 \\
18 \\
5\end{array}$ & $\begin{array}{r}46 \\
10 \\
4\end{array}$ & $\begin{array}{l}59 \cdot 0 \\
55 \cdot 5 \\
80 \cdot 0\end{array}$ & $\begin{array}{r}20 \\
11 \\
3\end{array}$ & $\begin{array}{r}11 \\
10 \\
2\end{array}$ & $\begin{array}{l}55 \cdot 0 \\
90 \cdot 9 \\
66 \cdot 7\end{array}$ \\
\hline & Female & $\begin{array}{l}\text { Non-RA } \\
\text { RA Intermediate } \\
\text { RA Probable and Definite }\end{array}$ & $\begin{array}{r}494 \\
166 \\
61\end{array}$ & $\begin{array}{r}416 \\
143 \\
44\end{array}$ & $\begin{array}{r}199 \\
75 \\
26\end{array}$ & $\begin{array}{l}47 \cdot 9 \\
52 \cdot 4 \\
59 \cdot 1\end{array}$ & $\begin{array}{l}64 \\
18 \\
10\end{array}$ & $\begin{array}{r}36 \\
12 \\
8\end{array}$ & $\begin{array}{l}56 \cdot 3 \\
66 \cdot 7 \\
80 \cdot 0\end{array}$ & $\begin{array}{r}14 \\
5 \\
7\end{array}$ & $\begin{array}{l}8 \\
2 \\
3\end{array}$ & $\begin{array}{l}57 \cdot 2 \\
40 \cdot 0 \\
42 \cdot 9\end{array}$ \\
\hline \multirow[t]{2}{*}{ Hips } & Male & $\begin{array}{l}\text { Non-RA } \\
\text { RA Intermediate } \\
\text { RA Probable and Definite }\end{array}$ & $\begin{array}{r}164 \\
75 \\
12\end{array}$ & $\begin{array}{r}137 \\
59 \\
8\end{array}$ & $\begin{array}{l}8 \\
8 \\
0\end{array}$ & $\begin{array}{r}5 \cdot 8 \\
13 \cdot 6 \\
0 \cdot 0\end{array}$ & $\begin{array}{r}11 \\
8 \\
3\end{array}$ & $\begin{array}{l}0 \\
2 \\
0\end{array}$ & $\underset{0}{0}+0$ & $\begin{array}{r}16 \\
8 \\
1\end{array}$ & $\begin{array}{l}9 \\
2 \\
0\end{array}$ & $\begin{array}{c}56 \cdot 3 * \\
25 \cdot 0 \\
0 \cdot 0\end{array}$ \\
\hline & Female & $\begin{array}{l}\text { Non-RA } \\
\text { RA Intermediate } \\
\text { RA Probable and Definite }\end{array}$ & $\begin{array}{r}178 \\
92 \\
46 \\
\end{array}$ & $\begin{array}{r}167 \\
81 \\
39\end{array}$ & $\begin{array}{r}16 \\
17 \\
6\end{array}$ & $\begin{array}{r}9 \cdot 6 \\
21 \cdot 0 \\
15 \cdot 4\end{array}$ & $\begin{array}{l}5 \\
4 \\
4\end{array}$ & $\begin{array}{l}0 \\
2 \\
1\end{array}$ & $\begin{array}{l}0 \\
50 \cdot 0 \\
25 \cdot 0\end{array}$ & $\begin{array}{l}6 \\
7 \\
3\end{array}$ & $\begin{array}{l}6 \\
5 \\
3\end{array}$ & $\begin{array}{l}100 \cdot 0^{*} \\
71 \cdot 5 \\
100 \cdot 0\end{array}$ \\
\hline \multirow[t]{2}{*}{$\begin{array}{l}\text { Ist } \\
\text { metatarso- } \\
\text { phalangeal }\end{array}$} & Male & $\begin{array}{l}\text { Non-RA } \\
\text { RA Intermediate } \\
\text { RA Probable and Definite }\end{array}$ & $\begin{array}{r}891 \\
172 \\
26\end{array}$ & $\begin{array}{r}739 \\
132 \\
16\end{array}$ & $\begin{array}{r}16 \\
18 \\
4\end{array}$ & $\begin{array}{r}2 \cdot 2 \\
13 \cdot 6 \\
25 \cdot 0\end{array}$ & $\begin{array}{r}118 \\
32 \\
8\end{array}$ & $\begin{array}{l}5 \\
2 \\
3\end{array}$ & $\begin{array}{r}4 \cdot 2 \\
6 \cdot 3 \\
37 \cdot 8\end{array}$ & $\begin{array}{r}34 \\
8 \\
2\end{array}$ & $\begin{array}{l}9 \\
5 \\
1\end{array}$ & $\begin{array}{l}26 \cdot 5 * * \\
62 \cdot 5 \\
50 \cdot 0\end{array}$ \\
\hline & Female & $\begin{array}{l}\text { Non-RA } \\
\text { RA Intermediate } \\
\text { RA Probable and Definite }\end{array}$ & $\begin{array}{r}897 \\
220 \\
74\end{array}$ & $\begin{array}{r}701 \\
151 \\
44\end{array}$ & $\begin{array}{l}27 \\
23 \\
17\end{array}$ & $\begin{array}{r}3 \cdot 8 \\
15 \cdot 2 \\
38 \cdot 6\end{array}$ & $\begin{array}{r}147 \\
56 \\
17\end{array}$ & $\begin{array}{r}19 \\
9 \\
6\end{array}$ & $\begin{array}{l}12 \cdot 9 \\
16 \cdot 1 \\
35 \cdot 3\end{array}$ & $\begin{array}{l}49 \\
13 \\
13\end{array}$ & $\begin{array}{r}13 \\
3 \\
3\end{array}$ & $\begin{array}{l}26 \cdot 5 \\
23 \cdot 1 \\
23 \cdot 1\end{array}$ \\
\hline
\end{tabular}

Probability of difference in proportion of pain between Osteo-arthrosis Grades 0-1 and 3-4: $*=<0 \cdot 05 \quad * *=<0 \cdot 01$. 


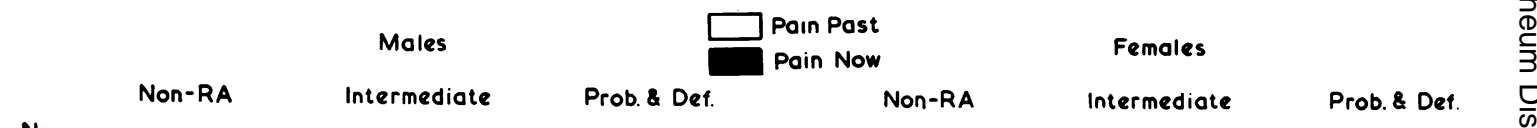

No.

$\sum \bar{N} 0 \quad \approx \sim$ N

$\approx \approx \approx$

요

$\stackrel{\circ}{\circ} \infty$

CARPOMETACARPAL
JOINTS
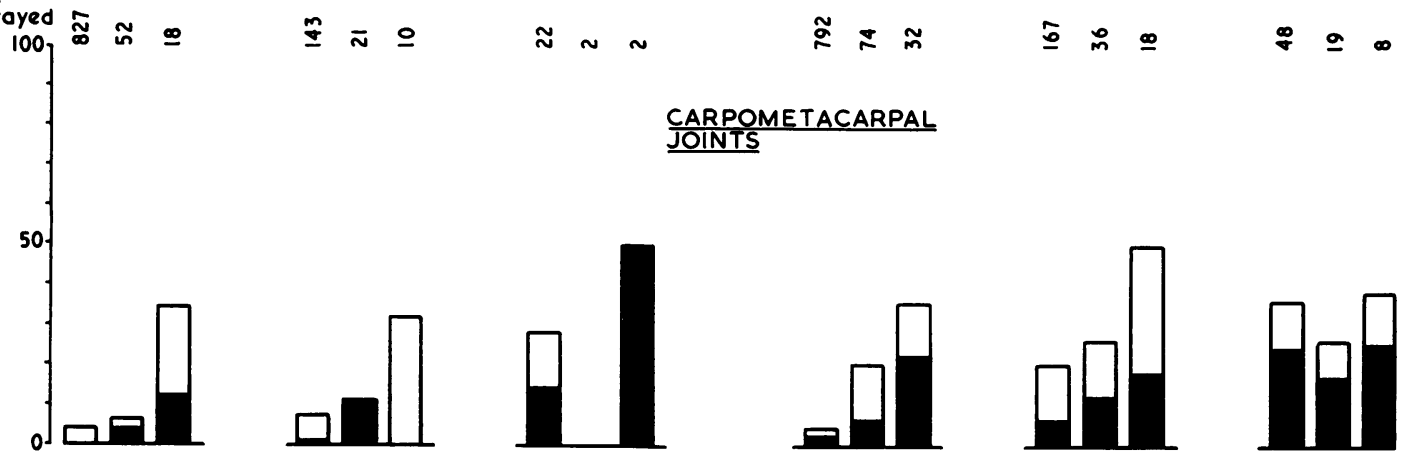

离


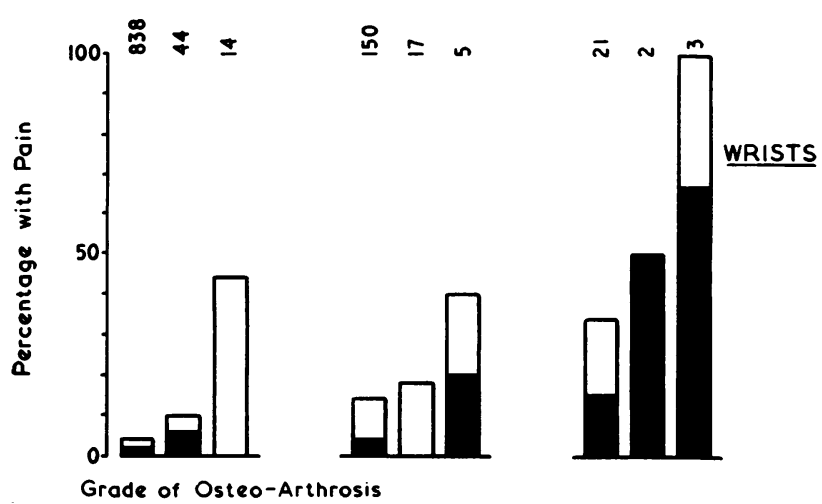

$\bar{\infty}$ ก

$\stackrel{\infty}{n}$

: $0 \mathrm{~m}$
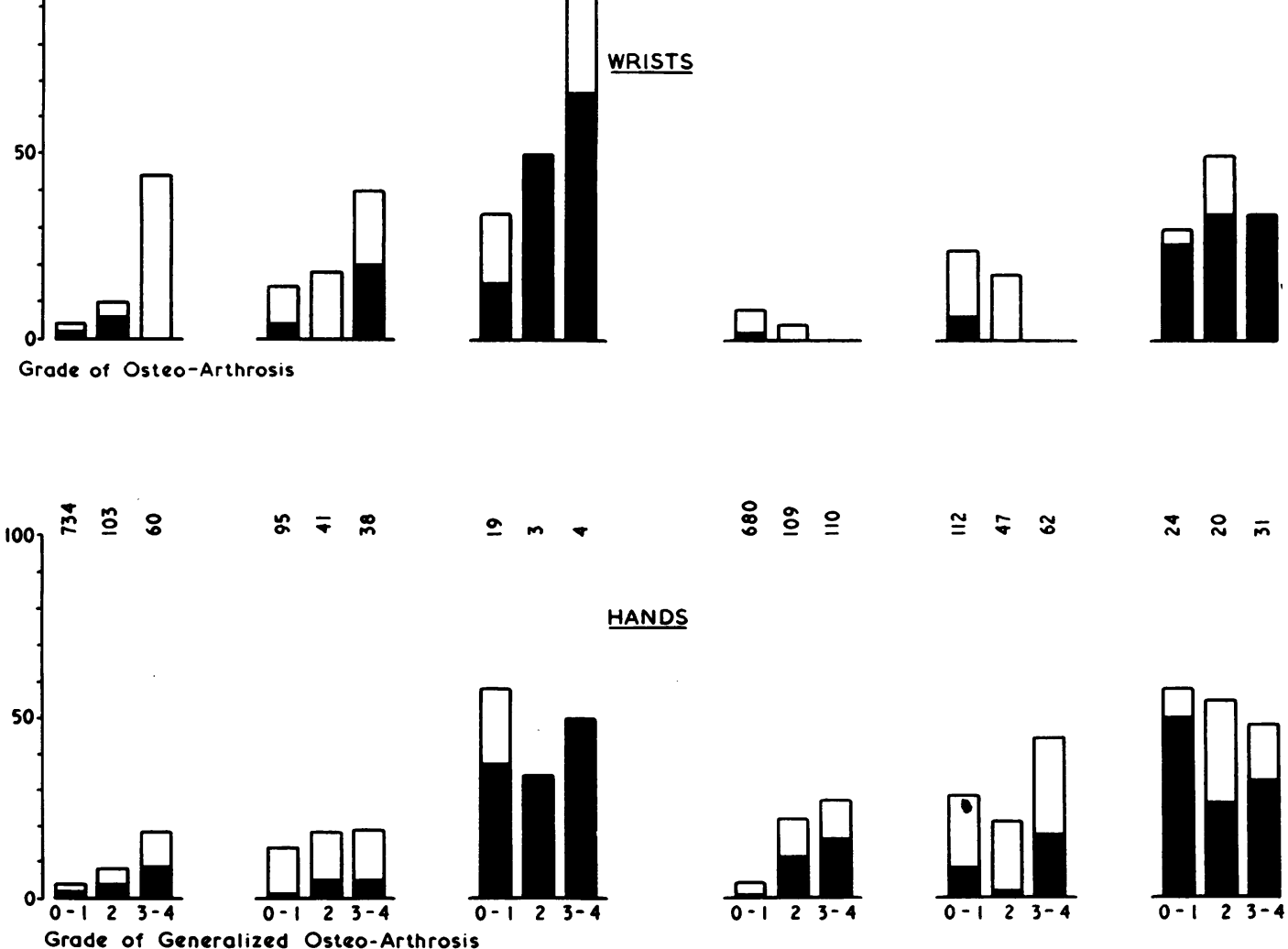

$\stackrel{\circ}{\circ}$ 인

$\cong \approx \approx$

$\sim$

\section{HANDS}

Fig. 3.-Relationship of grade of osteo-arthrosis of the hand and wrist to symptoms, by diagnosis and sex.
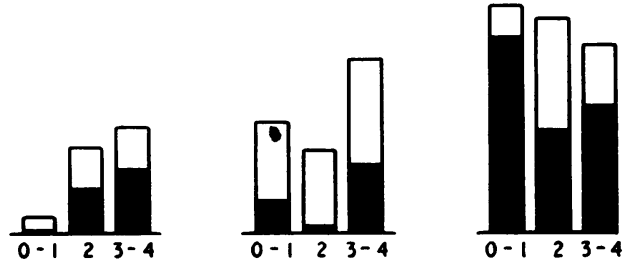

을

N

ก

స్ట

웅

$\stackrel{0}{\frac{0}{0}}$ 
was less definite. There was a significant association between Grade 3 to 4 osteo-arthrosis and pain in the neck, shoulder, and arms in the non-rheumatoid males, but the complaint rate was only double that found in the Grade 0 to 1 group (Table VII and Fig. 4). The pain was limited to the neck in some and to the shoulders in others, but was generally in the neck-shoulder-brachial distribution (C4-6). In the intermediate males there was a reverse trend but it was not significant. In female non-rheumatoids, the same trend was apparent as in the males. There was no relationship between osteo-arthrosis and symptoms in the female intermediate rheumatoids, but the relationship was significant at the 5 per cent. level in probable and definite rheumatoids if male and females were taken together.

Osteo-arthrosis of the Lumbar Spine.-This wal not associated with any increase of symptoms in the non-rheumatoid males (Table VII and Fig. 4 $\overrightarrow{\mathrm{F}^{*}}$

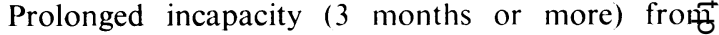
lumbar-sciatic pain - not shown in the Tables - was present in 19 per cent. of non-rheumatoid males wit Grade 3 to 4 osteo-arthrosis compared with 7 pe⿸丆口 cent. of those with Grade 0 to 1 but the associatio was not significant. In the rheumatoid males, ofp the other hand, there was significantly more pain $i \vec{\Phi}$ those with Grade 3 to 4 osteo-arthrosis. This 
difference may depend on the presence of a third cause of lumbar-sciatic pain. Lumbar disk degeneration, for example, which is known to be associated with lumbar-sciatic pain, was more frequent in all age groups in the rheumatoids with Grade 3 to 4 osteo-arthrosis than in those with Grade 0 to 1 osteo-arthrosis. No such association between disk degeneration and osteo-arthrosis was found in the non-rheumatoid males.

In females, there was no association between symptoms and osteo-arthrosis in either the rheumatoid or non-rheumatoid group.

There was no pain in the hip in those with Grade 2 osteo-arthrosis of that joint in the non-rheumatoid group (Table VII and Fig. 5, overleaf) but eight of them had knee pain, three without osteo-arthrosis of the knees. According to the age distribution, six would have been expected to have knee pain; this point will be discussed later. A feature of Grade 3 to 4 osteo-arthrosis of the hips was the high disability rate. A quarter of both males and females with this grade of change had lost 3 months or more from work because of hip pain. This applied equally in the rheumatoid and non-rheumatoid groups. Only 1 per cent. of those with Grade 0 to 1 osteo-arthrosis had such prolonged disability and these were mainly females with rheumatoid arthritis. Disability was particularly marked when both the hips and knees were affected.

Osteo-arthrosis of the Knees (Table VIII and Fig. 5).- The knees differed from the other joints in that symptoms were almost as frequent in the nonrheumatoid as in the rheumatoid group. The high complaint rate, 23 per cent. in males and 20 per cent. in females without osteo-arthrosis or rheumatoid arthritis, indicates either that some other cause of pain is important in the knee or that minor degrees of change are more difficult to recognize than in other joints. In some of those without osteoarthrosis or rheumatoid arthritis, the pain followed an injury to the knee, in others it appeared to be referred from the hip or spine. In most, however, the cause was undetermined. It is of interest that

TABLE VIII

RELATIONSHIP OF SYMPTOMS TO RADIOLOGICAL EVIDENCE OF OSTEO-ARTHROSIS IN THE KNEES IN PERSONS AGED 35+ YEARS IN THE LEIGH AND WENSLEYDALE ENLARGED SAMPLES

\begin{tabular}{|c|c|c|c|c|c|c|c|c|c|c|c|c|}
\hline \multirow{4}{*}{ Sex } & \multirow{4}{*}{ RA or Non-RA } & \multirow{4}{*}{$\underset{\substack{\text { Age } \\
\text { (yrs) }}}{\text { Arup }}$} & \multirow{4}{*}{$\begin{array}{l}\text { Total } \\
x \text { rayed }\end{array}$} & \multicolumn{9}{|c|}{ Grade of Osteo-arthrosis (Radiological) } \\
\hline & & & & \multicolumn{3}{|c|}{$0-1$} & \multicolumn{3}{|c|}{2} & \multicolumn{3}{|c|}{$3-4$} \\
\hline & & & & \multirow{2}{*}{$\begin{array}{c}\text { Total } \\
\text { No. }\end{array}$} & \multicolumn{2}{|c|}{ With Pain } & \multirow[b]{2}{*}{$\begin{array}{l}\text { Total } \\
\text { No. }\end{array}$} & \multicolumn{2}{|c|}{ With Pain } & \multirow{2}{*}{$\begin{array}{l}\text { Total } \\
\text { No. }\end{array}$} & \multicolumn{2}{|c|}{ With Pain } \\
\hline & & & & & No. & $\begin{array}{c}\text { Per } \\
\text { cent. of } \\
\text { Total }\end{array}$ & & No. & $\begin{array}{c}\text { Per } \\
\text { cent. of } \\
\text { Total }\end{array}$ & & No. & $\begin{array}{c}\text { Per } \\
\text { cent. of } \\
\text { Total }\end{array}$ \\
\hline \multirow{6}{*}{ Male } & \multirow[t]{2}{*}{ (a) Non-RA } & $\begin{array}{l}35-44 \\
45-54 \\
55-64 \\
65+ \\
\end{array}$ & $\begin{array}{r}162 \\
194 \\
103 \\
91 \\
\end{array}$ & $\begin{array}{r}153 \\
178 \\
74 \\
67 \\
\end{array}$ & $\begin{array}{l}27 \\
43 \\
16 \\
22 \\
\end{array}$ & $\begin{array}{l}17 \cdot 6 \\
24 \cdot 2 \\
21 \cdot 6 \\
32 \cdot 8\end{array}$ & $\begin{array}{r}8 \\
14 \\
22 \\
16 \\
\end{array}$ & $\begin{array}{l}3 \\
6 \\
7 \\
9 \\
\end{array}$ & $\begin{array}{l}37 \cdot 5 \\
42 \cdot 9 \\
31 \cdot 8 \\
56 \cdot 2\end{array}$ & $\begin{array}{l}1 \\
2 \\
7 \\
8\end{array}$ & $\begin{array}{l}1 \\
2 \\
3 \\
4\end{array}$ & $\begin{array}{r}100 \cdot 0 \\
100 \cdot 0 \\
42.9 \\
50 \cdot 0\end{array}$ \\
\hline & & Total & 550 & 472 & 108 & $22 \cdot 9$ & 60 & 25 & $41 \cdot 7$ & 18 & 10 & $55 \cdot 5^{*}$ \\
\hline & (b) RA Intermediate & Total & 145 & 103 & 32 & $31 \cdot 1$ & 36 & 18 & $50 \cdot 0$ & 6 & 5 & $83 \cdot 3$ \\
\hline & (c) RA Probable and Definite & Total & 24 & 18 & 10 & $55 \cdot 5$ & 6 & 5 & $83 \cdot 3$ & 0 & 二 & - \\
\hline & \multirow[t]{2}{*}{$(b)+(c)$} & $\begin{array}{l}35-44 \\
45-54 \\
55-64 \\
65+ \\
\end{array}$ & $\begin{array}{l}22 \\
39 \\
38 \\
70 \\
\end{array}$ & $\begin{array}{l}20 \\
36 \\
27 \\
38\end{array}$ & $\begin{array}{r}5 \\
14 \\
13 \\
10\end{array}$ & $\begin{array}{l}25 \cdot 0 \\
38 \cdot 9 \\
48 \cdot 1 \\
26 \cdot 3\end{array}$ & $\begin{array}{r}2 \\
3 \\
9 \\
28 \\
\end{array}$ & $\begin{array}{r}1 \\
2 \\
6 \\
14 \\
\end{array}$ & $\begin{array}{l}50 \cdot 0 \\
66 \cdot 7 \\
66 \cdot 7 \\
50 \cdot 0\end{array}$ & $\begin{array}{l}0 \\
0 \\
2 \\
4 \\
\end{array}$ & $\bar{z}$ & $\begin{array}{r}\overline{ } \\
100 \cdot 0 \\
75 \cdot 0\end{array}$ \\
\hline & & Total & 169 & 121 & 42 & $34 \cdot 7$ & 42 & 23 & $54 \cdot 3$ & 6 & 5 & $83 \cdot 3$ \\
\hline \multirow{6}{*}{ Female } & \multirow[t]{2}{*}{ (a) Non-RA } & $\begin{array}{l}35-44 \\
45-54 \\
55-64 \\
65+ \\
\end{array}$ & $\begin{array}{l}176 \\
168 \\
110 \\
112 \\
\end{array}$ & $\begin{array}{r}169 \\
146 \\
66 \\
57 \\
\end{array}$ & $\begin{array}{l}21 \\
34 \\
18 \\
15 \\
\end{array}$ & $\begin{array}{l}12 \cdot 4 \\
23 \cdot 3 \\
27 \cdot 3 \\
26 \cdot 3\end{array}$ & $\begin{array}{r}6 \\
18 \\
30 \\
35 \\
\end{array}$ & $\begin{array}{r}4 \\
6 \\
14 \\
15 \\
\end{array}$ & $\begin{array}{l}66 \cdot 7 \\
33 \cdot 3 \\
46 \cdot 7 \\
42 \cdot 9 \\
\end{array}$ & $\begin{array}{r}1 \\
4 \\
14 \\
20 \\
\end{array}$ & $\begin{array}{r}1 \\
3 \\
10 \\
17 \\
\end{array}$ & $\begin{array}{r}100.0 \\
75.0 \\
71.4 \\
85.0 \\
\end{array}$ \\
\hline & & Total & 566 & 438 & 88 & $20 \cdot 1$ & 89 & 39 & $43 \cdot 3$ & 39 & 31 & $79 \cdot 5^{* *}$ \\
\hline & (b) RA Intermediate & Total & 187 & 123 & 42 & $34 \cdot 1$ & 40 & 26 & $65 \cdot 0$ & 24 & 18 & $75 \cdot 0$ \\
\hline & (c) RA Probable and Definite & Total & 73 & 34 & 18 & $52 \cdot 9$ & 21 & 18 & $85 \cdot 7$ & 18 & 16 & $88 \cdot 9$ \\
\hline & \multirow[t]{2}{*}{$(b)+(c)$} & $\begin{array}{l}35-44 \\
45-54 \\
55-64 \\
65+ \\
\end{array}$ & $\begin{array}{r}32 \\
56 \\
60 \\
112 \\
\end{array}$ & $\begin{array}{l}28 \\
51 \\
37 \\
41 \\
\end{array}$ & $\begin{array}{l}12 \\
22 \\
15 \\
11 \\
\end{array}$ & $\begin{array}{l}42 \cdot 9 \\
43 \cdot 1 \\
40 \cdot 5 \\
26 \cdot 8 \\
\end{array}$ & $\begin{array}{r}3 \\
3 \\
15 \\
40 \\
\end{array}$ & $\begin{array}{r}1 \\
1 \\
11 \\
31\end{array}$ & $\begin{array}{l}33 \cdot 3 \\
33 \cdot 3 \\
73 \cdot 3 \\
77 \cdot 5 \\
\end{array}$ & $\begin{array}{r}1 \\
2 \\
8 \\
31 \\
\end{array}$ & $\begin{array}{r}1 \\
2 \\
8 \\
23\end{array}$ & $\begin{array}{r}100 \cdot 0 \\
100 \cdot 0 \\
100 \cdot 0 \\
74 \cdot 2\end{array}$ \\
\hline & & Total & 260 & 157 & 60 & $38 \cdot 2$ & 61 & 44 & $72 \cdot 2$ & 42 & 34 & $80 \cdot 9^{* *}$ \\
\hline
\end{tabular}

Probability of difference in proportion of pain between Osteo-arthrosis Grades 0-1 and 3-4: $*=<0 \cdot 05 * *=<0.01$. 


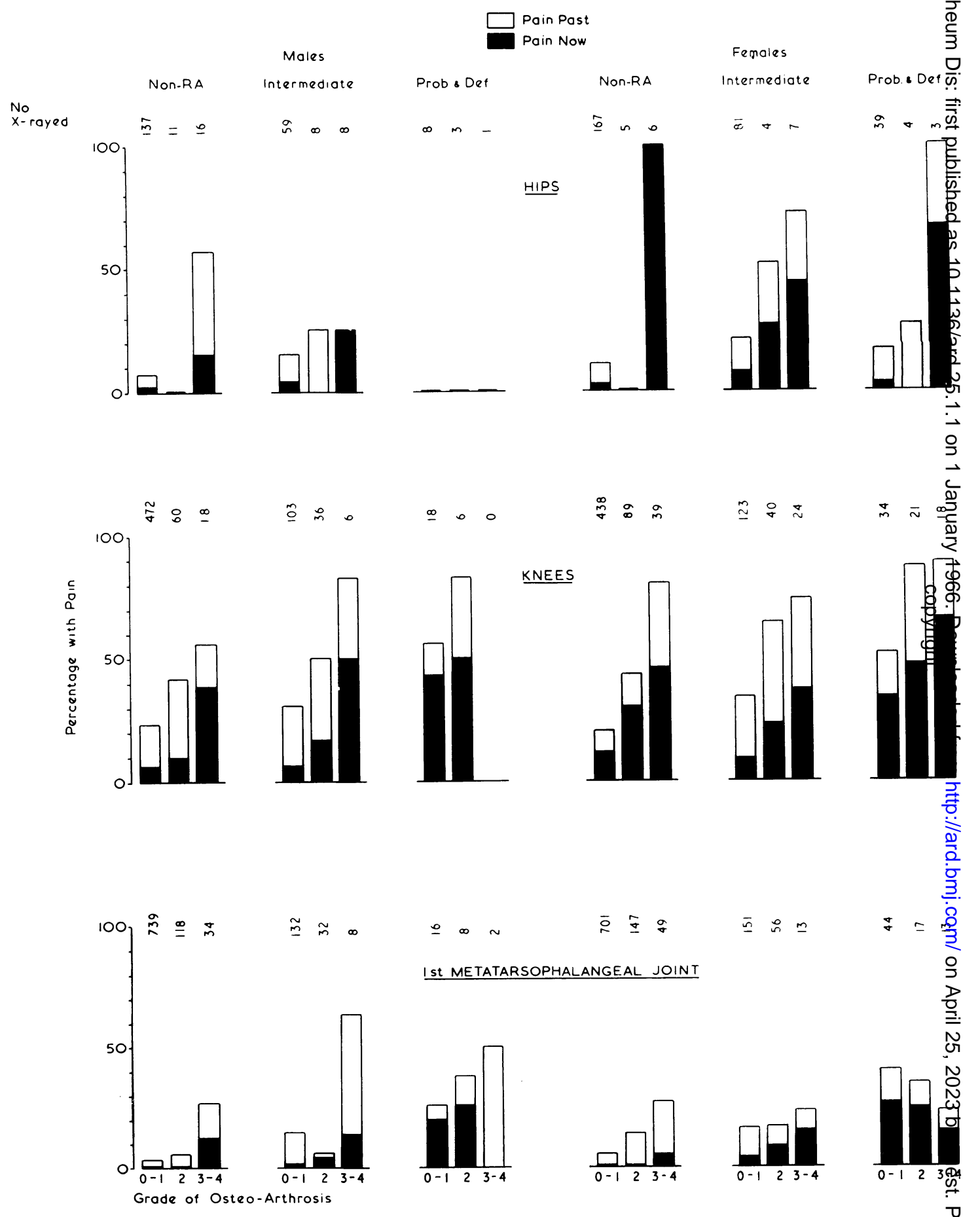

Fig. 5.- Relationship of grade of osteo-arthrosis of the hips, knees, and first metatarsophalangeal joint to symptoms, by diagnosis and s $\frac{\bar{Q}_{\bar{Q}}}{\bar{D}}$ 
only 6 per cent. of males and 7 per cent. of females in this category had pain actually at the time of the survey, compared with 39 per cent. and 46 per cent. respectively of those with Grade 3 to 4 osteoarthrosis. A feature of osteo-arthrosis in the knees was its relationship to loss of work from knee pain (Table IX). This showed a steeper gradient with increasing severity of osteo-arthrosis than simple pain, the proportion losing 3 months or more from knee pain rising from 1.5 to 8 per cent. in the nonrheumatoid males and from 1 to 5 per cent. in the nonrheumatoid females $(P<0.05)$. This also applied on a higher level in the rheumatoid groups, but was significant only in the males and females combined $(\mathrm{P}<0.05)$.

As might be expected, obesity had some influence not only on the prevalence of osteo-arthrosis in the knees but also on the occurrence of symptoms in those with osteo-arthrosis. The influence of obesity appeared to be greater in males, in whom it was significant in both the rheumatoid and non-rheumatoid groups $(P<0.05)$. In the females this influence was observed only in those without rheumatoid arthritis (Table $X$ and Fig. 6, overleaf). Obesity had also a significant influence on knee pain in males without osteo-arthrosis $(\mathrm{P}<0.05)$. It did not affect the frequency of pain in the hands, fingers, or thumbs in relation to generalized osteo-arthrosis in the hands (Fig. 7, overleaf).

There was little osteo-arthrosis in the tarsal and lateral metatarsophalangeal joints (not shown in the Tables). These demonstrated much the same relationship between osteo-arthrosis and symptoms as other joints but, whereas both males and females showed quite a marked association in the tarsal joints, this was limited to females in the case of the

TABLE IX

RELATIONSHIP OF MENTAL STABILITY TO KNEE PAIN AND DISABILITY IN THE LEIGH AND WENSLEYDALE ENLARGED SAMPLES

\begin{tabular}{|c|c|c|c|c|c|c|c|c|c|c|c|c|c|c|c|c|c|}
\hline \multirow{5}{*}{ Sex } & \multirow{5}{*}{$\begin{array}{l}\text { RA or } \\
\text { Non- } \\
\text { RA }\end{array}$} & \multirow{5}{*}{ Mental Stability } & \multirow{5}{*}{$\begin{array}{c}\text { Total } \\
x \text { rayed } \\
\text { Age } \\
35+\end{array}$} & \multicolumn{14}{|c|}{ Grade of Osteo-arthrosis (Radiological) } \\
\hline & & & & \multicolumn{7}{|c|}{$0-1$} & \multicolumn{7}{|c|}{$2-4$} \\
\hline & & & & \multirow{2}{*}{$\begin{array}{c}\text { Total } \\
\text { No. }\end{array}$} & \multirow[b]{2}{*}{ 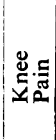 } & \multicolumn{2}{|c|}{$\underset{\text { Work }}{\text { Off }}$} & \multirow{2}{*}{ 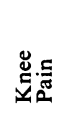 } & \multicolumn{2}{|c|}{ Off Work } & \multirow{2}{*}{$\begin{array}{c}\text { Total } \\
\text { No. }\end{array}$} & \multirow{2}{*}{ 离. } & \multicolumn{2}{|c|}{$\begin{array}{c}\text { Off } \\
\text { Work }\end{array}$} & \multirow[b]{2}{*}{ 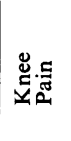 } & \multicolumn{2}{|c|}{ Off Work } \\
\hline & & & & & & $\begin{array}{c}1 \\
\text { wk } \\
+ \\
\end{array}$ & $\begin{array}{c}3 \\
\mathrm{mth} \\
+\end{array}$ & & $\begin{array}{c}1 \text { wk } \\
+\end{array}$ & $\begin{array}{c}3 \mathrm{mth} \\
+\end{array}$ & & & $\begin{array}{c}1 \\
\text { wk } \\
+\end{array}$ & $\begin{array}{c}3 \\
\text { mth } \\
+\end{array}$ & & $\begin{array}{c}1 \text { wk } \\
+\end{array}$ & $\begin{array}{c}3 \mathrm{mth} \\
+\end{array}$ \\
\hline & & & & \multicolumn{4}{|c|}{ Numbers } & \multicolumn{3}{|c|}{ Per cent. of Total } & \multicolumn{4}{|c|}{ Numbers } & \multicolumn{3}{|c|}{ Per cent. of Total } \\
\hline \multirow{6}{*}{ Male } & \multirow[t]{2}{*}{$\begin{array}{l}\text { Non- } \\
\text { RA }\end{array}$} & $\begin{array}{l}\text { Normal or Doubtful } \\
\text { Neurotic } \\
\text { Not stated or Psychotic }\end{array}$ & $\begin{array}{l}513 \\
24 \\
13\end{array}$ & $\begin{array}{r}446 \\
18 \\
8\end{array}$ & $\begin{array}{r}104 \\
3 \\
1\end{array}$ & $\begin{array}{r}10 \\
0 \\
1\end{array}$ & $\begin{array}{l}6 \\
0 \\
1\end{array}$ & $\begin{array}{l}23 \cdot 4 \\
16 \cdot 7 \\
12 \cdot 5\end{array}$ & $\begin{array}{c}2 \cdot 3 \\
0 \\
12 \cdot 5\end{array}$ & $\begin{array}{l}1 \cdot 4 \\
0 \\
12 \cdot 5\end{array}$ & $\begin{array}{r}67 \\
6 \\
5\end{array}$ & $\begin{array}{r}30 \\
3 \\
2\end{array}$ & $\begin{array}{l}4 \\
2 \\
0\end{array}$ & $\begin{array}{l}4 \\
2 \\
0\end{array}$ & $\begin{array}{l}44 \cdot 3 \\
50 \cdot 0 \\
40 \cdot 0\end{array}$ & $\begin{array}{c}6 \cdot 0 \\
33 \cdot 3 \\
0\end{array}$ & $\begin{array}{c}6 \cdot 0 \\
33 \cdot 3 \\
0\end{array}$ \\
\hline & & Total & 550 & 472 & 108 & 11 & 7 & $22 \cdot 9$ & $2 \cdot 3$ & $1 \cdot 5$ & 78 & 35 & 6 & 6 & $44 \cdot 9$ & $7 \cdot 7$ & $7 \cdot 7$ \\
\hline & \multirow{2}{*}{$\begin{array}{c}\text { RA } \\
\text { Inter- } \\
\text { mediate }\end{array}$} & $\begin{array}{l}\text { Normal or Doubtful } \\
\text { Neurotic } \\
\text { Not stated or Psychotic }\end{array}$ & $\begin{array}{r}134 \\
7 \\
4\end{array}$ & $\begin{array}{r}95 \\
5 \\
3\end{array}$ & $\begin{array}{r}28 \\
3 \\
1\end{array}$ & $\begin{array}{r}15 \\
2 \\
0\end{array}$ & $\begin{array}{l}7 \\
0 \\
0\end{array}$ & $\begin{array}{l}29 \cdot 5 \\
60 \cdot 0 \\
33 \cdot 3\end{array}$ & $\begin{array}{c}15 \cdot 8 \\
20 \cdot 0 \\
0\end{array}$ & $\begin{array}{l}7 \cdot 4 \\
0 \\
0\end{array}$ & $\begin{array}{r}39 \\
2 \\
1\end{array}$ & $\begin{array}{r}21 \\
1 \\
1\end{array}$ & $\begin{array}{r}12 \\
1 \\
1\end{array}$ & $\begin{array}{l}7 \\
1 \\
1\end{array}$ & $\begin{array}{l}53 \cdot 9 \\
50 \cdot 0 \\
100\end{array}$ & $\begin{array}{l}30 \cdot 8 \\
50 \cdot 0 \\
100\end{array}$ & $\begin{array}{l}17 \cdot 9 \\
50 \cdot 0 \\
100\end{array}$ \\
\hline & & Total & 145 & 103 & 32 & 17 & 7 & $31 \cdot 1$ & $16 \cdot 5$ & $6 \cdot 8$ & 42 & 23 & 14 & 9 & $54 \cdot 8$ & $33 \cdot 3$ & $21 \cdot 4$ \\
\hline & \multirow{2}{*}{$\begin{array}{c}\text { RA } \\
\text { Prob- } \\
\text { able } \\
\text { and De- } \\
\text { finite }\end{array}$} & $\begin{array}{l}\text { Normal or Doubtful } \\
\text { Neurotic } \\
\text { Not stated or Psychotic }\end{array}$ & $\begin{array}{r}24 \\
0 \\
0\end{array}$ & $\begin{array}{r}18 \\
0 \\
0\end{array}$ & $\frac{10}{-}$ & 工 & $\underline{4}$ & $\begin{array}{c}55 \cdot 6 \\
-\end{array}$ & $\stackrel{27 \cdot 8}{-}$ & $\stackrel{22 \cdot 2}{-}$ & $\begin{array}{l}6 \\
0 \\
0\end{array}$ & $\underline{5}$ & - & $\frac{2}{-}$ & $\begin{array}{c}83 \cdot 3 \\
- \\
\end{array}$ & $\begin{array}{l}50 \cdot 0 \\
-\end{array}$ & $\underline{33 \cdot 3}$ \\
\hline & & Total & 24 & 18 & 10 & 5 & 4 & $55 \cdot 6$ & $27 \cdot 8$ & $22 \cdot 2$ & 6 & 5 & 3 & 2 & $83 \cdot 3$ & $50 \cdot 0$ & $33 \cdot 3$ \\
\hline \multirow{6}{*}{ Female } & \multirow[t]{2}{*}{$\begin{array}{l}\text { Non- } \\
\text { RA }\end{array}$} & $\begin{array}{l}\text { Normal or Doubtful } \\
\text { Neurotic } \\
\text { Not stated or Psychotic }\end{array}$ & $\begin{array}{r}500 \\
60 \\
6\end{array}$ & $\begin{array}{r}390 \\
44 \\
4\end{array}$ & $\begin{array}{r}76 \\
12 \\
0\end{array}$ & $\begin{array}{l}8 \\
1 \\
-\end{array}$ & $\begin{array}{r}4 \\
0 \\
-\end{array}$ & $\begin{array}{c}19 \cdot 5 \\
27 \cdot 3 \\
0\end{array}$ & $\begin{array}{l}2 \cdot 1 \\
2 \cdot 3 \\
-\end{array}$ & $\begin{array}{l}1 \cdot 0 \\
0 \\
\end{array}$ & $\begin{array}{r}110 \\
16 \\
2\end{array}$ & $\begin{array}{r}61 \\
9 \\
0\end{array}$ & $\begin{array}{r}6 \\
2 \\
-\end{array}$ & $\begin{array}{r}5 \\
1 \\
-\end{array}$ & $\begin{array}{c}55 \cdot 5 \\
56 \cdot 3 \\
0\end{array}$ & $\begin{array}{r}5 \cdot 5 \\
12 \cdot 5 \\
-\end{array}$ & $\begin{array}{l}4 \cdot 5 \\
6 \cdot 3 \\
-\end{array}$ \\
\hline & & Total & 566 & 438 & 88 & 9 & 4 & $20 \cdot 1$ & $2 \cdot 1$ & 0.9 & 128 & 70 & 8 & 6 & $54 \cdot 7$ & $6 \cdot 3$ & $4 \cdot 7$ \\
\hline & \multirow{2}{*}{$\begin{array}{c}\text { RA } \\
\text { Inter- } \\
\text { mediate }\end{array}$} & $\begin{array}{l}\text { Normal or Doubtful } \\
\text { Neurotic } \\
\text { Not stated or Psychotic }\end{array}$ & $\begin{array}{r}161 \\
18 \\
8\end{array}$ & $\begin{array}{r}109 \\
10 \\
4\end{array}$ & $\begin{array}{r}37 \\
4 \\
1\end{array}$ & $\begin{array}{r}17 \\
0 \\
0\end{array}$ & $\begin{array}{l}6 \\
0 \\
0\end{array}$ & $\begin{array}{l}34 \cdot 0 \\
40 \cdot 0 \\
25 \cdot 0\end{array}$ & $\begin{array}{c}15 \cdot 6 \\
0 \\
0\end{array}$ & $\begin{array}{l}5 \cdot 5 \\
0 \\
0\end{array}$ & $\begin{array}{r}52 \\
8 \\
4\end{array}$ & $\begin{array}{r}37 \\
4 \\
3\end{array}$ & $\begin{array}{r}11 \\
1 \\
2\end{array}$ & $\begin{array}{l}8 \\
0 \\
1\end{array}$ & $\begin{array}{l}71 \cdot 2 \\
50 \cdot 0 \\
75 \cdot 0\end{array}$ & $\begin{array}{l}21 \cdot 1 \\
12 \cdot 5 \\
50 \cdot 0\end{array}$ & $\begin{array}{c}15 \cdot 4 \\
0 \\
25 \cdot 0\end{array}$ \\
\hline & & Total & 187 & 123 & 42 & 17 & 6 & $34 \cdot 1$ & $13 \cdot 8$ & $4 \cdot 9$ & 64 & 44 & 14 & 9 & $68 \cdot 8$ & $21 \cdot 9$ & $14 \cdot 1$ \\
\hline & \multirow{2}{*}{$\begin{array}{c}\text { RA } \\
\text { Prob- } \\
\text { able } \\
\text { and De- } \\
\text { finite }\end{array}$} & $\begin{array}{l}\text { Normal or Doubtful } \\
\text { Neurotic } \\
\text { Not stated or Psychotic }\end{array}$ & $\begin{array}{r}69 \\
1 \\
3\end{array}$ & $\begin{array}{r}33 \\
0 \\
1\end{array}$ & $\frac{18}{0}$ & $\underline{-}$ & $\underline{6}$ & $\frac{54 \cdot 6}{0}$ & $\stackrel{24 \cdot 2}{-}$ & $\stackrel{18 \cdot 2}{-}$ & $\begin{array}{r}36 \\
1 \\
2\end{array}$ & $\begin{array}{r}31 \\
1 \\
2\end{array}$ & $\begin{array}{r}14 \\
1 \\
1\end{array}$ & $\begin{array}{r}12 \\
0 \\
0\end{array}$ & $\begin{array}{l}86 \cdot 2 \\
100 \\
100\end{array}$ & $\begin{array}{l}38 \cdot 9 \\
100 \\
50 \cdot 0\end{array}$ & $\begin{array}{c}33 \cdot 4 \\
0 \\
0\end{array}$ \\
\hline & & Total & 73 & 34 & 18 & 8 & 6 & $52 \cdot 9$ & $23 \cdot 5$ & $17 \cdot 7$ & 39 & 34 & 16 & 12 & $87 \cdot 2$ & $41 \cdot 0$ & $30 \cdot 8$ \\
\hline
\end{tabular}


TABLE $X$

RELATIONSHIP OF OBESITY TO KNEE PAIN AND RADIOLOGICAL EVIDENCE OF OSTEO-ARTHROSIS IN THE KNEE IN PERSONS AGED $35+$ YRS

IN THE LEIGH AND WENSLEYDALE ENLARGED SAMPLES

\begin{tabular}{|c|c|c|c|c|c|c|c|c|c|}
\hline \multirow{4}{*}{ Sex } & \multirow{4}{*}{ RA or Non-RA } & \multirow{4}{*}{ Obesity } & \multirow{4}{*}{ 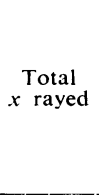 } & \multicolumn{6}{|c|}{ Grade of Osteo-arthrosis Knee (Radiological) } \\
\hline & & & & \multicolumn{3}{|c|}{$0-1$} & \multicolumn{3}{|c|}{$2-4$} \\
\hline & & & & \multirow{2}{*}{$\begin{array}{l}\text { Total } \\
\text { No. }\end{array}$} & \multicolumn{2}{|c|}{ With Pain } & \multirow{2}{*}{$\begin{array}{c}\text { Total } \\
\text { No. }\end{array}$} & \multicolumn{2}{|c|}{ With Pain } \\
\hline & & & & & No. & $\begin{array}{l}\text { Per cent. } \\
\text { of Total }\end{array}$ & & No. & $\begin{array}{l}\text { Per ces? } \\
\text { of Totad }\end{array}$ \\
\hline \multirow{6}{*}{ Male } & \multirow[t]{2}{*}{ Non-RA } & $\begin{array}{l}\text { Absent } \\
\text { Present } \\
\text { Not stated } \\
\end{array}$ & $\begin{array}{r}419 \\
131 \\
0\end{array}$ & $\begin{array}{l}370 \\
102 \\
\end{array}$ & $\begin{array}{l}76 \\
32 \\
\end{array}$ & $\begin{array}{c}20 \cdot 5 \\
31 \cdot 4 \\
-\end{array}$ & $\begin{array}{l}49 \\
29 \\
-\end{array}$ & $\begin{array}{l}15 \\
20 \\
-\end{array}$ & $\begin{array}{r}30 \cdot 6 \sqrt{\mathrm{D}} \\
69 \cdot 0 \\
-\mathrm{N}\end{array}$ \\
\hline & & Total & 550 & 472 & 108 & $22 \cdot 9$ & 78 & 35 & $44.9 \overrightarrow{0}$ \\
\hline & \multirow[t]{2}{*}{ RA Intermediate } & $\begin{array}{l}\text { Absent } \\
\text { Present } \\
\text { Not stated }\end{array}$ & $\begin{array}{r}110 \\
35 \\
0 \\
\end{array}$ & $\begin{array}{l}81 \\
22 \\
- \\
\end{array}$ & $\begin{array}{r}25 \\
7 \\
-\end{array}$ & $\begin{array}{r}30 \cdot 8 \\
31 \cdot 8 \\
- \\
\end{array}$ & $\begin{array}{r}29 \\
13 \\
- \\
\end{array}$ & $\begin{array}{l}12 \\
11 \\
\end{array}$ & $\begin{array}{r}41 \cdot 4-\vec{\omega} \\
84 \cdot 6 \vec{\omega} \\
-\sigma\end{array}$ \\
\hline & & Total & 145 & 103 & 32 & $31 \cdot 1$ & 42 & 23 & $54 \cdot 8 \stackrel{2}{\underline{n}}$ \\
\hline & \multirow[t]{2}{*}{ RA Probable and Definite } & $\begin{array}{l}\text { Absent } \\
\text { Present } \\
\text { Not stated }\end{array}$ & $\begin{array}{r}16 \\
8 \\
0 \\
\end{array}$ & $\begin{array}{r}13 \\
5 \\
- \\
\end{array}$ & $\begin{array}{r}7 \\
3 \\
- \\
\end{array}$ & $\begin{array}{r}53 \cdot 9 \\
40 \cdot 0 \\
- \\
\end{array}$ & $\begin{array}{r}3 \\
3 \\
-\end{array}$ & $\begin{array}{r}2 \\
3 \\
-\end{array}$ & $\begin{array}{r}66 \cdot \pi \\
100 \cdot 00 \\
-\quad-7 \\
\end{array}$ \\
\hline & & Total & 24 & 18 & 10 & $55 \cdot 5$ & 6 & 5 & $83 \cdot 3$ \\
\hline \multirow{6}{*}{ Female } & \multirow[t]{2}{*}{ Non-RA } & $\begin{array}{l}\text { Absent } \\
\text { Present } \\
\text { Not stated }\end{array}$ & $\begin{array}{r}344 \\
220 \\
2 \\
\end{array}$ & $\begin{array}{r}304 \\
134 \\
0 \\
\end{array}$ & $\begin{array}{r}55 \\
33 \\
\end{array}$ & $\begin{array}{c}18 \cdot 1 \\
24 \cdot 6 \\
--\end{array}$ & $\begin{array}{r}40 \\
86 \\
2\end{array}$ & $\begin{array}{r}16 \\
54 \\
0\end{array}$ & $\begin{array}{l}40 \cdot 0^{5} \\
62 \cdot 8 \\
0.0 \\
\alpha\end{array}$ \\
\hline & & Total & 566 & 438 & 88 & $20 \cdot 1$ & 128 & 70 & $54 \cdot 7$ ב \\
\hline & \multirow[t]{2}{*}{ RA Intermediate } & $\begin{array}{l}\text { Absent } \\
\text { Present } \\
\text { Not stated }\end{array}$ & $\begin{array}{r}114 \\
72 \\
1 \\
\end{array}$ & $\begin{array}{r}84 \\
38 \\
1\end{array}$ & $\begin{array}{r}27 \\
15 \\
0\end{array}$ & $\begin{array}{c}33 \cdot 3 \\
39 \cdot 5 \\
0\end{array}$ & $\begin{array}{r}30 \\
34 \\
0\end{array}$ & $\begin{array}{l}20 \\
24 \\
-\end{array}$ & $\begin{array}{l}66 \cdot \frac{0}{2} \\
-\frac{6}{0}\end{array}$ \\
\hline & & Total & 187 & 123 & 42 & $34 \cdot 1$ & 64 & 44 & 68ి \\
\hline & \multirow[t]{2}{*}{ RA Probable and Definite } & $\begin{array}{l}\text { Absent } \\
\text { Present } \\
\text { Not stated } \\
\end{array}$ & $\begin{array}{r}36 \\
35 \\
2 \\
\end{array}$ & $\begin{array}{r}22 \\
11 \\
1 \\
\end{array}$ & $\begin{array}{r}11 \\
6 \\
1 \\
\end{array}$ & $\begin{array}{r}50 \cdot 0 \\
54 \cdot 6 \\
100 \cdot 0 \\
\end{array}$ & $\begin{array}{r}14 \\
24 \\
1 \\
\end{array}$ & $\begin{array}{r}13 \\
20 \\
1 \\
\end{array}$ & 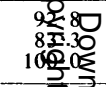 \\
\hline & & Total & 73 & 34 & 18 & $52 \cdot 9$ & 39 & 34 & $87 \cdot 20$ \\
\hline
\end{tabular}

lateral metatarsophalangeal joints. In view of the small numbers with osteo-arthrosis in this joint group, no significance can be attached to this.

Morning Stiffness.-It was not possible to grade morning stiffness according to replies from different observers as in the survey by Cobb and others (1957), since in most instances the question was asked by only one physician. The relationship of morning stiffness to pain has been studied in the hands and knees (Table XI, overleaf; Fig. 6, opposite; Fig. 7, overleaf).

It was not possible fully to confirm the findings of Cobb and his colleagues. Symptoms were rather more frequent in those with morning stiffness than in those without in the non-rheumatoid group regardless of whether osteo-arthrosis was present or not. This applied in the intermediate group in both sexes in relation to osteo-arthrosis in the knee, and in the males also in relation to generalized osteoarthrosis in the hands, but in the rheumatoid group symptoms were not consistently more frequent in those with morning stiffness than in those witho粮t. The difference in the proportion with pain in the presence and absence of morning stiffness reachesat least as low as the 5 per cent. probability level in the various male categories, except in the nenrheumatoid males with osteo-arthrosis Grade 2 to 4 where there are only fifteen subjects with mornmg stiffness. In female non-rheumatoids and beth rheumatoid categories combined, the differe reaches at least as low as $<0.05$ probability onlygin osteo-arthrosis of the knee Grade 0 to 1 but not at Grade 2 to 4 .

\section{Other Causes of Symptoms}

Injury and Symptoms. - The importance of traufiga as a cause of osteo-arthrosis has been stressed in the past. Stecher (1948) observed that Heberdeeg's nodes often resulted from injury. In a survey Leigh the knee was the most frequent site Off traumatic osteo-arthrosis (Kellgren and Lawrence, 1958). We have therefore made an analysis of 
Moles

Females
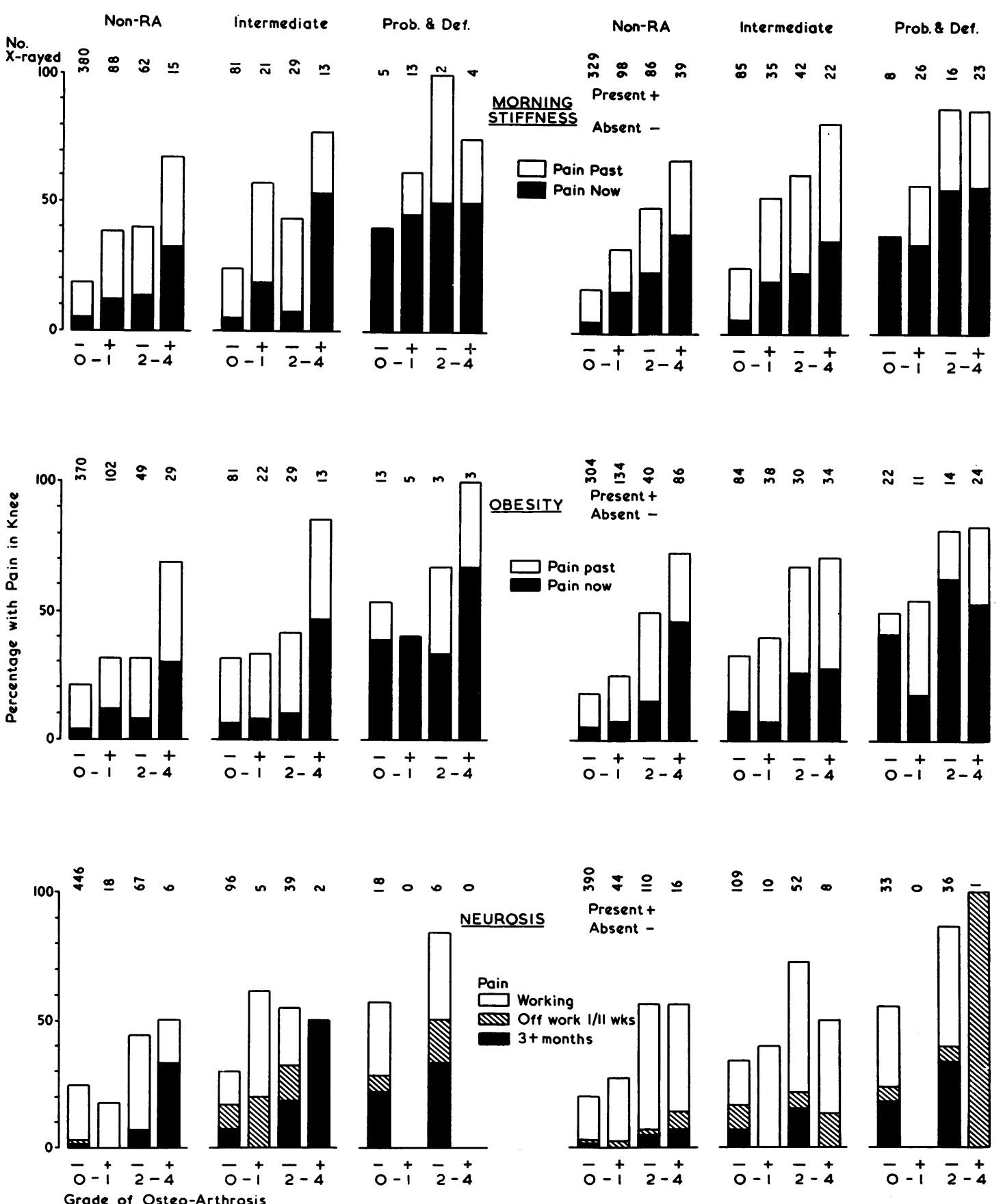

Fig. 6.-Relationship of morning stiffness, obesity, and neurosis to knee pain in osteo-arthrosis of the knees, by grade of os teo-arthrosis diagnosis, and sex. 


\begin{tabular}{|c|c|c|c|c|c|c|c|c|c|}
\hline \multirow{4}{*}{ Sex } & \multirow{4}{*}{ RA or Non-RA } & \multirow{4}{*}{$\begin{array}{l}\text { Morning } \\
\text { Stiffness }\end{array}$} & \multirow{4}{*}{$\begin{array}{c}\text { Total } \\
x \text { rayed }\end{array}$} & \multicolumn{6}{|c|}{ Grade of Osteo-arthrosis Knee (Radiological) } \\
\hline & & & & \multicolumn{3}{|c|}{$0-1$} & \multicolumn{3}{|c|}{$2-4$} \\
\hline & & & & \multirow{2}{*}{$\begin{array}{c}\text { Total } \\
\text { No. }\end{array}$} & \multicolumn{2}{|c|}{ With Pain } & \multirow{2}{*}{$\begin{array}{l}\text { Total } \\
\text { No. }\end{array}$} & \multicolumn{2}{|c|}{ With Pain $\underline{\underline{\sigma}}$} \\
\hline & & & & & No. & $\begin{array}{l}\text { Per cent. } \\
\text { of Total }\end{array}$ & & No. & $\begin{array}{l}\text { Per cent } \\
\text { of Tota }\end{array}$ \\
\hline \multirow{6}{*}{ Male } & \multirow[t]{2}{*}{ Non-RA } & $\begin{array}{l}\text { Absent } \\
\text { Present } \\
\text { Not stated }\end{array}$ & $\begin{array}{r}442 \\
103 \\
5\end{array}$ & $\begin{array}{r}380 \\
88 \\
4\end{array}$ & $\begin{array}{r}72 \\
33 \\
3\end{array}$ & $\begin{array}{l}19 \cdot 0 \\
37 \cdot 5 \\
75 \cdot 0\end{array}$ & $\begin{array}{r}62 \\
15 \\
1\end{array}$ & $\begin{array}{r}25 \\
10 \\
0\end{array}$ & 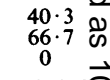 \\
\hline & & Total & 550 & 472 & 108 & $22 \cdot 9$ & 78 & 35 & $44 \cdot 9 \stackrel{.}{=}$ \\
\hline & \multirow[t]{2}{*}{ RA Intermediate } & $\begin{array}{l}\text { Absent } \\
\text { Present } \\
\text { Not stated }\end{array}$ & $\begin{array}{r}110 \\
34 \\
1\end{array}$ & $\begin{array}{r}81 \\
21 \\
1\end{array}$ & $\begin{array}{r}19 \\
12 \\
1\end{array}$ & $\begin{array}{r}23 \cdot 5 \\
57 \cdot 2 \\
100 \cdot 0\end{array}$ & $\begin{array}{r}29 \\
13 \\
0\end{array}$ & $\begin{array}{l}13 \\
10 \\
-\end{array}$ & 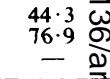 \\
\hline & & Total & 145 & 103 & 32 & $31 \cdot 1$ & 42 & 23 & $54 \cdot 8 \stackrel{0}{N}$ \\
\hline & \multirow[t]{2}{*}{ RA Probable and Definite } & $\begin{array}{l}\text { Absent } \\
\text { Present } \\
\text { Not stated } \\
\end{array}$ & $\begin{array}{r}7 \\
17 \\
0\end{array}$ & $\begin{array}{r}5 \\
13 \\
0\end{array}$ & $\begin{array}{r}2 \\
8 \\
-\end{array}$ & $\begin{array}{c}40 \cdot 0 \\
61 \cdot 5 \\
- \\
\end{array}$ & $\begin{array}{l}2 \\
4 \\
0\end{array}$ & $\begin{array}{r}2 \\
3 \\
-\end{array}$ & $\begin{array}{r}100.0 \\
75.0 \\
-\end{array}$ \\
\hline & & Total & 24 & 18 & 10 & $55 \cdot 6$ & 6 & 5 & $83 \cdot 3$ 工 \\
\hline \multirow{6}{*}{ Female } & \multirow[t]{2}{*}{ Non-RA } & $\begin{array}{l}\text { Absent } \\
\text { Present } \\
\text { Not stated }\end{array}$ & $\begin{array}{r}415 \\
137 \\
14\end{array}$ & $\begin{array}{r}329 \\
98 \\
11\end{array}$ & $\begin{array}{r}55 \\
32 \\
1\end{array}$ & $\begin{array}{r}16 \cdot 7 \\
32 \cdot 7 \\
9 \cdot 1\end{array}$ & $\begin{array}{r}86 \\
39 \\
3\end{array}$ & $\begin{array}{r}43 \\
26 \\
1\end{array}$ & $\begin{array}{l}50 \cdot 0 \bar{c} \\
66 \cdot 7 \stackrel{\text { ڤ్ }}{33 \cdot 3}\end{array}$ \\
\hline & & Total & 566 & 438 & 88 & $20 \cdot 1$ & 128 & 70 & $54 \cdot 7 \stackrel{\text { ฏू }}{2}$ \\
\hline & \multirow[t]{2}{*}{ RA Intermediate } & $\begin{array}{l}\text { Absent } \\
\text { Present } \\
\text { Not stated }\end{array}$ & $\begin{array}{r}127 \\
57 \\
3\end{array}$ & $\begin{array}{r}85 \\
35 \\
3\end{array}$ & $\begin{array}{r}22 \\
19 \\
1\end{array}$ & $\begin{array}{l}25 \cdot 9 \\
54 \cdot 3 \\
33 \cdot 3\end{array}$ & $\begin{array}{r}42 \\
22 \\
0\end{array}$ & $\begin{array}{l}26 \\
18 \\
-\end{array}$ & 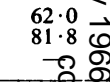 \\
\hline & & Total & 187 & 123 & 42 & $34 \cdot 2$ & 64 & 44 & $68 \overline{8}$ \\
\hline & \multirow[t]{2}{*}{ RA Probable and Definite } & $\begin{array}{l}\text { Absent } \\
\text { Present } \\
\text { Not stated } \\
\end{array}$ & $\begin{array}{r}24 \\
49 \\
0\end{array}$ & $\begin{array}{r}8 \\
26 \\
0\end{array}$ & $\begin{array}{r}3 \\
15 \\
\end{array}$ & $\begin{array}{l}37 \cdot 5 \\
57 \cdot 7 \\
-\end{array}$ & $\begin{array}{r}16 \\
23 \\
0 \\
\end{array}$ & $\begin{array}{l}14 \\
20 \\
-\end{array}$ & 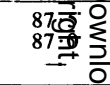 \\
\hline & & Total & 73 & 34 & 18 & $53 \cdot 0$ & 39 & 34 & $87 \cdot 2$ ฉ \\
\hline
\end{tabular}

these two joints to determine the relationship between injury and symptoms. Of the 32 males with Grade 3 to 4 osteo-arthrosis of the distal interphalangeal joints, three had pain; one of these had no history of injury, one had a history of fractured $2 \mathrm{nd}$, 3rd, and 4th digits but on the side which was free of pain, and the third gave a history of injury only to the chest. Of the 135 with Grade 2 osteo-arthrosis, eleven had pain; five of these gave a history of injury to the affected finger but no indication of its relationship to the painful episodes. In four injury had probably preceded the osteo-arthrosis; one on the other hand had an injury to the right 2 nd and 3rd digits but had bilateral pain in the distal interphalangeal joints, and one had been blown up in the first war but had no pain in the fingers till 1952 . The remainder gave no history of a related injury. There was thus no direct relationship of injury to the onset of symptoms in any non-rheumatoid males with osteo-arthrosis of the distal interphalangeal joints. Nor did any of the non-rheumatoid females with osteo-arthrosis of these joints give a history of injury to the fingers.

A history of injury to the knee joint is not umcommon especially in males. Of the ten males with Grade 3 to 4 osteo-arthrosis in the knees a id symptoms, one had had an injury to the legs, of which the left had been amputated, but the duration of symptoms in the right knee and the date of injury was not given. Another had a history of injury go the right knee but had osteo-arthrosis and symptoms in both knees. A third gave a history of a cartilage operation to the affected knee 33 years previously but had had no injury before his recent onset $8 \mathrm{ff}$ symptoms. A fourth gave a history of a dislocatest cartilage but not in the knee which was causing pato A fifth had had trouble with his knees since wounds 36 years before. A sixth had twisted bis right knee but had osteo-arthrosis and symptoms कूn both knees. It would thus seem that injuries of the type likely to be remembered are not responsible for the onset of symptoms of osteo-arthrosis. THifs 


\section{OSTEO-ARTHROSIS}
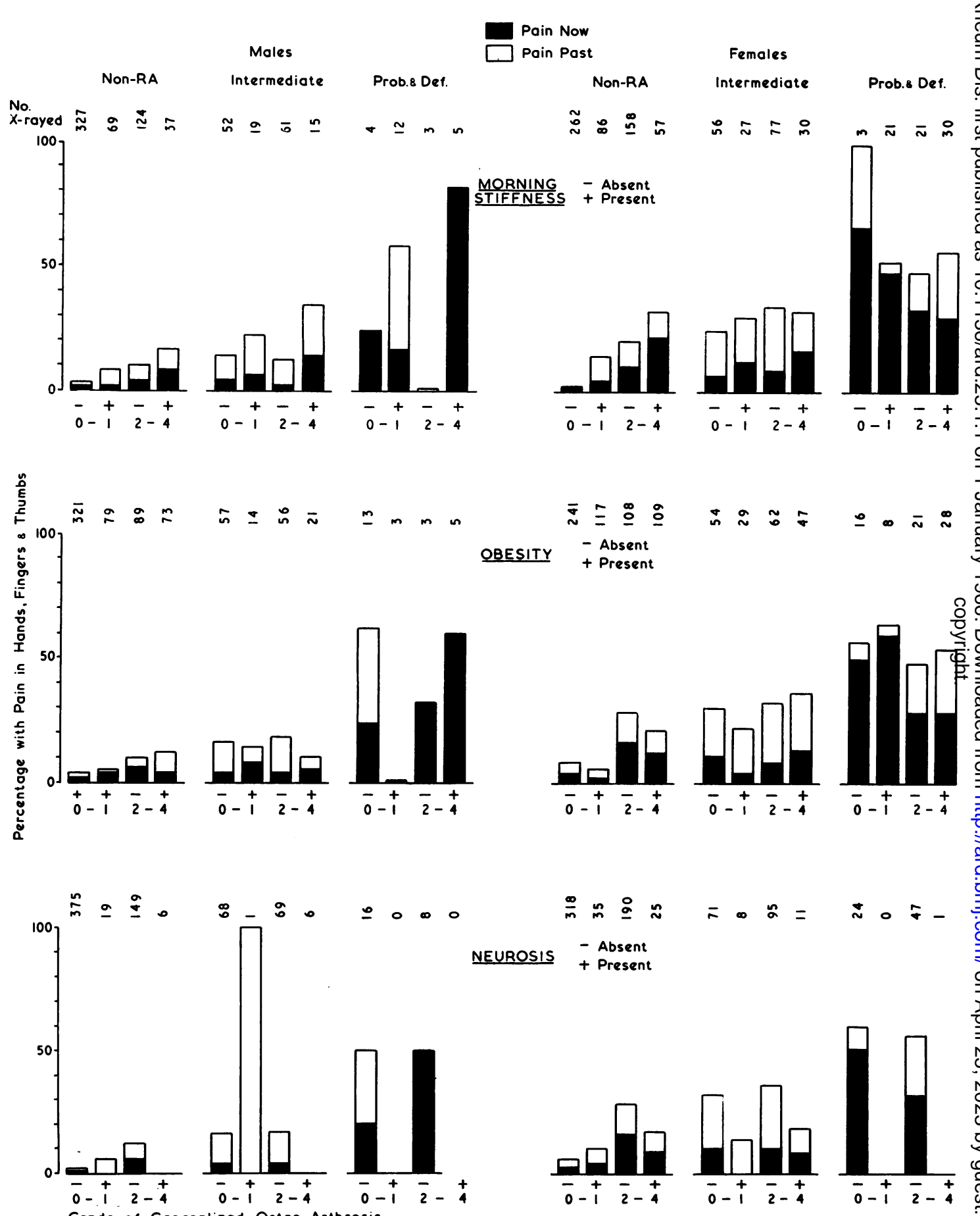

$\frac{\infty}{m} \stackrel{\infty}{\infty} \approx$
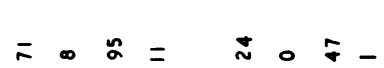

$$
\text { NEUROSIS - Absent }
$$
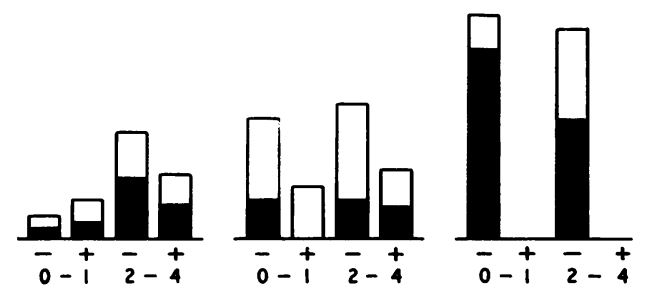

Grode of Generalized Osteo-Arthrosis Fig. 7.-Relationship of morning stiffness, obesity, and neurosis to hand and finger pain in generalized osteo-arthrosis of the hands, by grade ofteo-arthrosis, diagnosis, and sex. 
does not exclude minor trauma as a cause of the painful episodes associated with osteo-arthrosis.

Neurosis and Complaint Rate.-It is often taken for granted that mentally unstable persons complain more and therefore are more likely to have symptoms in association with any organic disease which may be present. To determine whether this applied in osteo-arthrosis, each respondent in the Leigh and Wensleydale surveys was given a grading for mental stability according to the following code:

$$
\begin{aligned}
& 0=\text { stable } \\
& 1=\text { doubtful } \\
& 2=\text { neurotic } \\
& 3=\text { psychotic }
\end{aligned}
$$

This grading had previously been checked against a series of psychometric tests and found to correlate satisfactorily (Heron and Braithwaite, 1953). There was no evidence that the complaint rate in osteoarthrosis was affected by mental instability in either sex (Table IX and Figs 6 and 7). In each group the proportion losing work from rheumatic complaints was roughly the same in the neurotic and mentally stable persons. Loss of work from other complaints may have been greater in the less stable individuals but this was not determined. The neurotic individuals with osteo-arthrosis of the knees lost slightly more work in the non-rheumatoid group, but this did not approach significance and the total complaint rate was much the same as in the stable and unstable persons with osteo-arthrosis. Pain in the hands was actually less in the neurotic individuals with osteo-arthrosis of the hands than in the mentally stable. Indeed none of the neurotic males with $x$ ray evidence of generalized osteo-arthrosis in the hands had symptoms (Fig. 7).

Damp Housing. - The number of damp houses in the population sample in Leigh was small. Only nineteen of the respondents there lived, at the time of the survey, in houses designated by the Council as very damp, i.e. having defective subsoil drainage or structural defects. There were seventy respondents living in damp houses in Wensleydale; these were houses in which the damp was severe enough to justify demolition but they were probably less damp than those defined by the stricter criteria in Leigh. In the damp houses the frequency of knee pain in those with $x$ ray changes of osteo-arthrosis was much the same as in the dry houses and, though there was more pain at the time of the survey and more loss of work in those living in the damp houses, the numbers involved were too small for any conclusions to be drawn. The only significant finding was a greater prevalence of knee pain in the nonrheumatoid females living in damp houses. This was not due to their having more osteo-arthrosic change or to a difference in age distribution, and $\frac{D^{\circ}}{\widehat{D}}$ there was no evidence that those with osteo-arthrosis $\frac{\bigodot}{3}$ had moved out of the damp houses. In view of the large number of houses which were described as damp in Wensleydale, it must be considered whether less strict criteria were used to determine dampness $\stackrel{\oplus}{\stackrel{\rho}{+}}$ in that area. When Leigh and Wensleydale were analysed separately, it was found that the complaint $\frac{\bar{G}}{\bar{m}}$ rate of osteo-arthrosis of the knees from those $\frac{\bar{\sigma}}{\overrightarrow{0}}$ living in damp houses in Leigh was higher than that $\stackrel{\Phi}{\varrho}$ from those in the dry houses. The numbers, though they supported previous findings in Leigh, were too small for any conclusions to be drawn.

Bone Cysts.-These are a prominent feature of $\overrightarrow{\vec{\omega}}$ osteo-arthrosis. They have been shown to be associated with defects in the articular cartilage and $\overline{0}$ subchondral bone, and it has been suggested that on they are formed when synovial fluid is forced through under pressure during movement of the joint. If the $\vec{\circ}$ fluid content of the cysts is under pressure at such times it would seem reasonable to expect that pain $\vec{c}$ might result. Bone cysts are encountered most frequently in the metacarpophalangeal joints of the hands. In the Leigh and Wensleydale surveys, 46 males and 31 females had cysts in the neighbourhood $\overrightarrow{0}$ of these joints. Pain was not significantly morg frequent in those with cysts than in those withoue and the numbers were too small to confirm the relationship to symptoms in those with oste arthrosis. Bone cysts are a frequent finding in osteo-arthrosis of the hips. Of the persons with $\frac{\varnothing}{\varnothing}$ Grade 3 to 4 osteo-arthrosis of the hips in the $\cong$ Leigh and Wensleydale samples, 21 had bone cysts, $\overrightarrow{\vec{O}}$ of these eleven had had pain in the hips. Of the 3 twenty without bone cysts, fourteen had had pain. Thus it would seem unlikely that bone cysts are an important cause of pain in the hips, nor was there an increased complaint rate in association with bone cysts in other joints studied.

\section{Discussion}

The increasing importance of osteo-arthrosis in the community should first be stressed. Even in the 을 past decade there has been an appreciable rise in the $D$ proportion of the population over the age of $55 \stackrel{\text {. }}{=}$ years. It is in this age range that osteo-arthrosis N begins to produce incapacity.

From the analyses which have been presented, it $N$ is clear that, except in the lumbar apophyseal joints, the presence of osteo-arthrosis carries a definite predisposition to symptoms in the affected region $\varrho$ and that this predisposition is related to the degree $\mathbb{D}$ of $x$ ray change. The most striking association between the $x$ ray changes of osteo-arthrosis and 0 symptoms was found in the 1st metatarsophalangeal 
joints, where the frequency of symptoms rose from 2 to 26 per cent. with increasing $x$ ray change in males. It is evident, however, that osteo-arthrosis is a predisposing factor rather than a cause of symptoms, since most of those with the disease had no symptoms in the corresponding region. On comparing Figs 1 to 7 and the supporting Tables, the reader should bear in mind that, in our sample comprising the adult population of any age, the proportion of people with symptoms in osteoarthrosis Grade 0 to 1 is mostly based on sizeable total numbers (700 to 800 in the non-rheumatoid and 100 to 200 in the rheumatoid intermediate group) so that the upper 5 per cent. confidence limits lie, in the non-rheumatoid, only 1 to 4 per cent. and, in the rheumatoid intermediate group, only 6 to 9 per cent. above the percentages shown, whereas the total number of subjects with osteo-arthrosis Grade 2 (mild but definite) is rarely above 100 and that of subjects with osteo-arthrosis Grade 3 to 4 (moderate to severe) is rarely above thirty. Occasionally it is, even in the above groups, considerably smaller, so that one has to reckon here with much wider confidence limits. Thus, in the Tables for the various joint groups, the 5 per cent. confidence limits for the higher grades of osteo-arthrosis are not uncommonly 20 per cent. or more below or above the percentages shown. This applies particularly to males, in whom (as shown in Table II) moderate or severe osteoarthrosis is less frequent than in females. We felt it was interesting enough to show the picture in full detail for the small probable and definite rheumatoid group, although the numbers involved here in the various sub-groups were often small.

In spite of these natural deficiences of our sample, certain features repeat themselves with remarkable regularity in many of the joint groups examined. Except for the spinal, sacro-iliac, and knee joints, which will have to be considered separately, the proportion with pain lies, in the non-rheumatoids with osteo-arthrosis Grade 0 to 1 , usually well below 10 per cent., particularly in males, and there was a fairly smooth rise, seldom to less than 20 per cent. in those with osteo-arthrosis Grade 3 to 4, again with somewhat lower proportions in males than females. The individual differences are significant at the 5 or 1 per cent. levels of probability as indicated in the Tables by single and double asterisks and the repetition of similar findings in many joint groups makes them the more impressive.

The hip joint seems to stand out somewhat in our data, with no pain at osteo-arthrosis Grade 2 in male and female non-rheumatoids, but, considering that the upper 5 per cent. confidence limit of 0 per cent. in a sample of sixteen (taking males and fe- males together) is 21 per cent., this finding by itself $\overrightarrow{\widetilde{\sigma}}$ is statistically unremarkable and becomes interesting $\frac{5}{3}$ only in connexion with the tentative medical explanation given above. On adding those with Grade 2 osteo-arthrosis to those with Grade 3 to 4 , $\overrightarrow{\bar{B}}$ the combined group, again taking male and female $\stackrel{\rho}{?}$ non-rheumatoids together, still differs from Grade 0 to 1 at the 1 per cent. level of significance.

Though the total rheumatoid groups are con- $\frac{\sqrt{2}}{8}$ siderably smaller than the non-rheumatoid, this- 2 according to the differing age distribution-mainly $\overbrace{}^{\circ}$ affects osteo-arthrosis Grade 0 to 1 , while the other $\vec{\circ}$ osteo-arthrosic sub-groups are usually-but not without exception-more equal in size. This gives $\vec{\omega}$ some strength to our further comparison. Indeed, it is easy to see from Figs 2 to 5 that, in practically all the joint groups, there is a rise in the proportion of of symptoms with the grade of osteo-arthrosis and that this closely parallels the rise in the non- rheumatoid groups, though from a higher starting $\frac{\circ}{2}$ point at osteo-arthrosis Grade 0 to 1 , which rarely lies lower than 10 per cent. in the intermediate and 30 per cent. in the probable and definite group. Again, significant differences between the lowest and $\stackrel{\varrho}{2}$ the highest grades of osteo-arthrosis have been $\overrightarrow{-}$ indicated in the Tables, taking, in this calculation, $\mathscr{\odot}$ intermediate, probable, and definite cases togethes. Considering the numbers involved, it is not sut prising that the rheumatoids do not show as man significant differences as the non-rheumatoids, and the male rheumatoids less than the females, but the general trend seems undisputable.

The two spinal regions examined, the cervical and $\overrightarrow{\overrightarrow{0}}$ lumbar, present a picture very different from that of 3 the other joint groups. Both male and female nonrheumatoids show, in osteo-arthrosis Grade 0 to 1 , a complaint rate of nearly 40 per cent. in the cervical spine and about 50 per cent. in the lumbar spine. This does not appear very different from the findings in the rheumatoid group. Since the osteo-arthrosis Grade 0 to 1 sub-group is particularly large in both 의 spinal regions, separate sortings (not shown in the Table and Figure) were made for osteo-arthrosis 5 Grade 0 and Grade 1, but the findings in each of $D$ these two grades were very much alike. There was? some increase of complaint rate in non-rheumatoids $N$ with Grade 1 osteo-arthrosis, but this was comparatively slight, whilst in the rheumatoids the picture at higher grades of osteo-arthrosis was more $\omega$ irregular. This is not surprising, considering the small numbers involved. A clear rise in the numbere with symptoms from increasing severity of osteo- $\mathbb{\Phi}$ arthrosis, however, came out in considering the ${ }^{-}$ lumbar spine in the male rheumatoids, probably $\frac{0}{2}$ because of a closer linkage than in the non-rheuma- 
toid males with lumbar disk degeneration, which started at an earlier age in the rheumatoids than in the non-rheumatoids. It seems likely that disk degeneration plays a major role in the causation of pain in the spinal regions. This subject will be dealt with more fully in another paper.

The factors which determine whether symptoms arise are not easily determined. Cobb's suggestion that most of the symptoms of osteo-arthrosis are due to rheumatoid arthritis is not supported by this study. Those with rheumatoid arthritis had on the whole more joint pain whether osteo-arthrosis was present or not, but there was no evidence of any synergic effect, and indeed there was in most joint groups a closer relationship between the grade of osteo-arthrosis and symptoms in the non-rheumatoid than in the rheumatoid subjects. In those with both diseases, the effect appears to be purely additive. If this is so, it would be expected that those joints, such as the metacarpophalangeal and cervical apophyseal, which are frequently affected in rheumatoid arthritis, would show less correlation between osteo-arthrosis and symptoms in females whether overt rheumatoid arthritis is present or not. This is in fact the case. Cobb based his conclusions mainly on an association between morning stiffness and the complaint rate due to osteo-arthrosis. When the osteo-arthrosis complaint rate in those with and without morning stiffness is compared in the Leigh and Wensleydale populations, it is evident that the rate is increased in those with stiffness but is less affected by morning stiffness than by osteo-arthrosis. Most osteoarthrosic subjects, when asked about morning stiffness, stated that their stiffness was increased by any type of rest and was not particularly severe in the mornings.

There was no evidence that the complaint rate due to osteo-arthrosis was different in males and females, except possibly in the distal interphalangeal joints of the fingers, where, in the absence of rheumatoid arthritis, it rose from 1 to 25 per cent. in females with increasing severity of osteo-arthrosis but only from 1 to 9 per cent. in males This may be due to the greater number of affected distal interphalangeal joints in females who more often have a generalized form of the disease, whereas in males the condition is more often traumatic and affects a single joint.

The absence of any correlation between $x$-ray changes and symptoms in the lumbar spine is not surprising, since the effect of osteophyte formation on the apophyseal joints may well be to stabilize the spine rather than to diminish its stability. In general, the relationship of $x$-ray changes to symptoms appears to be determined mainly by the natural mobility of the joint. This is well illu- 귱 strated in the fingers, where the association of $\frac{\mathbb{C}}{3}$ symptoms with $x$-ray changes is greater in the proximal than in the distal interphalangeal joints.

The present data do not permit a definite conclusion as to the cause of painful episodes in osteo- $\stackrel{D}{\stackrel{D}{+}}$ arthrosis. Overt trauma, though a frequent cause of the disease, seems to have little effect on the com-흘 plaint rate in those with pre-existing disease. $\frac{\bar{\rho}}{\sigma}$ Mechanical factors must play an important part, as $\underset{\complement}{\Phi}$ shown by the effect of obesity on the knees and by the striking difference in complaint rate between $\vec{P}$ those with Grade 2 and with Grade 3 to 4 osteo-? arthrosis. The main difference between these two $\vec{\omega}$ grades is the degree of wear of the articular cartilage, $\stackrel{\circ}{\circ}$ which is negligible in the Grade 2 but appreciable in those with Grade 3 change or above. The degree of trauma which is required to produce ligamentous strain in such unstable joints may well be so slight ${ }^{-}$ as to be forgotten by the respondent. Moreover,음 the relationship to trauma may be direct. LloydRoberts (1953) found that fragments from the degenerate articular surfaces, presumably detached? by trauma are taken up by the synovial membrane and give rise to an inflammatory response and fibrosis. The shortening of the capsule which fol- $\vec{\bullet}$ lows may, he thinks, give rise to pain on movemegt. According to Trueta (1954), there is an associated hypervascularity of juxta-articular bone which mey also be a source of pain.

The effect of damp is not well demonstrated in this survey, possibly because of the small number of ${ }_{0}^{\mathbb{D}}$ really damp houses to be found in a sample of this $\overrightarrow{\vec{A}}$ size. It would seem likely that the criteria for 3 dampness were less rigid in Wensleydale, and this may have obscured a real effect. It is perhaps of interest that, in the original Leigh survey, when allo houses classified as damp by the sanitary inspectoro were included, there was little difference in the prevalence of clinical osteo-arthrosis and disk degeneration, whereas when only very damp housesô (i.e. those with defective subsoil drainage or structural defect) were included the difference was more윽 marked. We consider that the harmful effect of $>$ damp housing must remain in doubt.

The association of symptoms with bone cysts must be considered unproven. It would seem. likely that these cysts are an important cause of pain $\sim$ in osteo-arthrosis, though they may sometimes give rise to pain independently of the presence of theo arthrosis.

The symptomatology of generalized osteo-arthro- $\frac{\text { }}{\Phi}$ sis has not been discussed in this paper, as it is to be? analysed in detail in a later communication dealing 0 specifically with the generalized forms of the disease. $\frac{\vec{\Phi}}{\mathbb{D}}$ 


\section{Summary}

A combined urban-rural population in the North of England was questioned about musculo-skeletal pain and loss of work, and was submitted to a clinical examination of the joints and to a routine series of joint $x$ rays. A sample of blood was tested for rheumatoid factor by the sheep-cell agglutination test. The $x$ rays were read without knowledge of the history or clinical state.

After standardization of the adult population for age and sex by the unweighted mean, the prevalence of osteo-arthrosis was found to be 52 per cent. in males and 51 per cent. in females. Five or more joint groups were affected in 9 per cent. of males and 12 per cent. of females, the proportion increasing rapidly with age to a maximum of 37 per cent. in males and 49 per cent. in females aged 65 and over. When minimal disease was excluded, the prevalence of osteo-arthrosis was 19 per cent. in males and 22 per cent. in females, and five or more joint groups were affected in 0.5 per cent. of males and 1.8 per cent. of females. Standardization to the 1961 population of England and Wales gave slightly lower prevalences in the males.

On the basis of the clinical, radiological, and serological findings, the population was divided into a rheumatoid and a non-rheumatoid group. In the non-rheumatoid group there was a significant correlation between $x$-ray changes of osteo-arthrosis and the frequency of symptoms in the corresponding distribution. This was present in all joints except those of the lumbar spine and was only marginal in the cervical spine. In the rheumatoid groups there was a higher rate of symptoms, but the increase of complaints with severity of osteo-arthrosis was no greater than in the non-rheumatoid group. Morning stiffness was associated with an increase of symptoms in the hands and knees in the nonrheumatoid group regardless of whether osteoarthrosis was present or not.

Obesity was associated with an increase of pain in the knees in persons with osteo-arthrosis but had little influence in those without.

Persons with osteo-arthrosis who had been classified as neurotic did not have a higher complaint rate than those not so classified, nor did those with a history of injury.

Persons with osteo-arthrosis living in damp houses did not have significantly more than the expected proportion of symptoms, but the numbers in very damp houses were small.

Symptoms were only slightly more when bone cysts were present in the metacarpophalangeal joints, but showed no relationship to symptoms in the hip joints.
We wish to express our thanks to Prof. J. H. Kellgren for permission to use his $x$-ray readings of the hand films and for his advice and criticism. We are indebted to Mr. H. Clusky, Sanitary Inspector to the Leigh Town Council, and to Mr. K. Jolley, of the Hawes (Wensleydale) Rural Council, for providing information on the damp houses in their areas.

For the statistical tables appearing in this paper a compiler consisting of "Atlas Autocode" and a number of inter-related routines for the "Analysis of Surveys" by D. Y. Downham, J. McKay, and D. Rutovitz and a guide to its use by the Manchester University Department of Social and Preventive Medicine (unpublished communication) were used. One of the authors (FB) received from D. Y. Downham some personal guidance in the application of the compiler. Our thanks are due to this team for their valuable assistance and to Manchester University for access to the Atlas Computer.

\section{REFERENCES}

Anderson, J. A. D., and Duthie, J. J. R. (1963). Ann. rheum. Dis., 22, 401.

- - - and Moody, B. P. (1962). Ibid., 21, 342.

Bremner, J. M. (1961). Ibid., 20, 149.

Cobb, S., Merchant, W. R., and Rubin, T. (1957). J. chron. Dis., 5, 197.

Heron, A., and Braithwaite, D. (1953). Brit. J. industr. Med., $10,27$.

Kellgren, J. H. (1961). Brit. med. J., $2,1$.

- and Lawrence, J. S. (1952). Brit. J. industr. Med. 9, 197.

(1957). Ann. rheum. Dis., 16, 494.

(1958). Ibid., 17, 388.

, and Aitken-Swan, J. (1953). Ibid., 12, 5.

, - , and Bier, F. (1963). Ibid., 22, 237.

and Moore, R. (1952). Brit. med.J., 1, 181.

Lawrence, J. S. (1955). Brit. J. industr. Med., 12, 249. and Bennett, P. H. (1960). Ann. rheum. Dis., 19, 20.

, Dingwall-Fordyce, I., and Molyneux, M. (1966). Brit. J. industr. Med., 23, 42.

Graaff, R. de, and Laine, V. A. I. (1963). In

"The Epidemiology of Chronic Rheumatism", p. 98, ed. J. H. Kellgren, M. R. Jeffrey, and J. Ball. Blackwell, Oxford.

Lloyd-Roberts, G. C. (1953). J. Bone Jt Surg., 35B, 627.

Mason, R. M. (1956). Ann. phys. Med., 3, 143.

Stecher, R. M. (1948). Ann. rheum. Dis., 7, 1.

Trueta, J. (1954). Ann. roy. Coll. Surg. Engl., 15, 174.

\section{L'ostéo-arthrose.-Sa fréquence dans la population et le rapport entre les symptômes et les altérations radiologiques. \\ RÉSUMÉ}

On interrogea une population combinée urbaine et rurale au Nord d'Angleterre sur la douleur musculosquélettique et la perte du temps de travail et on la soumit à un examen clinique des articulations et à une série systématique de radiographies. On préléva du sang pour la réaction de Waaler-Rose et pour y rechercher le facteur rhumatoide. On interpréta les radiographies sans connaître les antécédents ou l'état clinique des sujets.

Après avoir ajusté sans biais les chiffres de la population adulte, ayant égard à l'âge et au sexe, à la dis- 
tribution normale standardisée, on trouva que 52 pour cent des hommes et 51 pour cent des femmes furent atteints d'ostéo-arthrose. Cinq ou plus groupes d'articulations furent atteintes en 9 pour cent des hommes et 12 pour cent des femmes. Cette proportion augmenta rapidement avec l'âge pour atteindre un maximum de 37 pour cent pour les hommes et 49 pour cent pour les femmes après l'âge de 64 ans. Lorsqu'on exlut la lésion "minime", on trouve une fréquence d'ostéoarthrose de 19 pour cent chez des hommes et de 23 pour cent chez des femmes; l'atteinte d'un minimum de cinq groupes d'articulations s'observa alors en 0,5 pour cent des hommes et en 1,8 pour cent des femmes. Lorsqu'on standardise la distribution pour la faire correspondre à celle de la population anglaise et galloise de l'an 1961, on trouve la fréquence un peu plus basse chez les hommes.

Selon les résultats de l'examen clinique, radiologique et sérologique, la population fut divisée en rhumatoïde et non-rhumatoïde. Dans le groupe non-rhumatoïde il y eut une correlation significative entre les signes radiographiques d'ostéo-arthrose et la fréquence des symptômes dans la distribution correspondante. L'ostéo-arthrose fut présente dans toutes les articulations sauf celles de la colonne lombaire et ne fut que marginale dans la colonne cervicale. Dans le groupe rhumatoïde, les symptômes furent plus fréquents, mais cette fréquence, liée à la sévérité de l'ostéo-arthrose, ne fut pas plus haute que celle dans le groupe non-rhumatoïde. L'enraidissement matinal fut associé à l'augmentation des symptômes aux mains et aux genoux dans le groupe non-rhumatoïde, indépendamment de l'existence de l'ostéo-arthrose.

L'obésité fut associée à l'augmentation de la douleur aux genoux chez des personnes atteintes d'ostéoarthrite, mais non pas chez les autres.

Les personnes atteintes d'ostéo-arthrose et désignées comme "névrosées" n'accusèrent pas plus de symptômes que les autres; il en fut de même pour les accidentés.

Les personnes atteintes d'ostéo-arthrose qui habitaient dans une maison humide n'accusèrent guère plus de symptômes, mais le nombre de maisons très humides était petit.

Il y eut un peu plus de symptômes en présence des kystes articulaires métacarpophalangiennes, sans rapport toutefois aux symptômes articulaires de la hanche.

\section{Osteoartrosis.- Su frecuencia en la población y las relaciones entre los síntomas y las alteraciones radiológicas}

Sumario

Se interrogó una población combinada rural y urbana del Norte de Inglaterra sobre el dolor músculo- esquelético y ausencias del trabajo y se la sometió a uñ examen clínico de las articulaciones y a una serie sistemática de radiografías. Se recogió sangre para lä reacción de Waaler-Rose y para buscar el factof reumatoide. Se interpretaron las radiografías sir conocer la historia o la condición clínica de los sujetos.

Después de haber estandarizado sin bies la distribuciò de la población adulta, tomando en cuenta la edad y ef sexo, se halló que un 52 por ciento de los hombres y urc 51 por ciento de las mujeres tuvieron osteoartrosis은 Cinco o más grupos de articulaciones fueron afectadosf en un 9 por ciento de los hombres y un 12 por ciento d las mujeres. Esta proporción aumentó rapidamente con? la edad para llegar a su máximo del 37 por ciento en loš hombres y del 49 por ciento en las mujeres de más de64 años. Cuando se excluye la lesión "minimal" se observa la osteoartrosis en un 19 por ciento de losi hombres y en un 23 por ciento de las mujeres y lfw implicación de cinco o más grupos de articulacione ocurre en un 0,5 por ciento de los hombres y en un 1,8 po 5 ciento de las mujeres. Cuando se estandardiza laई cifras para hacerlas corresponder a las de la poblaciórọ inglesa y galesa de 1961, se halla una frecuencia algo menor en los hombres.

Según los resultados del examen clínico, radiológico 오 serológico, la población fué dividida en grupos reu= matoide y non-reumatoide. En el grupo non-reumatoide hubo una correlación significativa entre las alteracioneș radiográficas de osteoartrosis y la frecuencia de los síntomas en la distribuciòn correspondiente. Esto se viôّ en todas las articulaciones excepto en las de la columna lumbar y marginalmente en la columna cervical. En edo grupo reumatoide los síntomas fueron más frecuenfes pero esta frecuencia, ligada a la severidad deolá osteoartrosis, no fué mayor que la en el grupo nÆ̣̂n reumatoide. La rigidez matinal fué asociada corêée aumento de los síntomas en las manos y las rodillas en ${ }^{+} \mathrm{eP}$ grupo non-reumatoide, independientemente de existencia de la osteoartrosis.

La obesidad fué asociada al aumento del dolor en $1 \nsubseteq$ rodilla en personas con osteoartrosis, pero no lo fué en los demás.

Las personas con osteoartrosis clasificadas como neuróticas no tuvieron más síntomas que los demás; lơ mismo se aplica a los con una historia de accidente.

Personas con osteoartrosis viviendo en casas húmedaఖ no acusaron significativamente más síntomas, pero $\bar{\Phi}$ número de casas muy húmedas fué pequeño.

Hubo algo más síntomas en presencia de quistẹ metacarpofalangeos, pero no hubo tal relación co: síntomas articulares de la cadera. 